\title{
EIGENFUNCTIONS OF THE FOURIER TRANSFORM WITH SPECIFIED ZEROS
}

\author{
AHRAM S. FEIGENBAUM ${ }^{\dagger}$, PETER J. GRABNER* ${ }^{*}$, \\ AND DOUGLAS P. HARDIN ${ }^{\dagger}$
}

\section{INTRODUCTION}

The sphere packing problem is one of those mathematical problems which are easy to state and notoriously difficult to solve. A sphere packing $\mathcal{P}$ of $\mathbb{R}^{d}$ is a collection of congruent non-overlapping balls. Its (upper) density

$$
\Delta(\mathcal{P})=\limsup _{r \rightarrow \infty} \frac{\operatorname{vol}_{d}(\mathcal{P} \cap B(\mathbf{0}, r))}{\operatorname{vol}_{d}(B(\mathbf{0}, r))}
$$

is the amount of space covered by $\mathcal{P}$ (here $B(\mathbf{0}, r)$ denotes the euclidean ball of radius $r$ centered at $\mathbf{0}$; $\operatorname{vol}_{d}$ is the $d$-dimensional Lebesgue measure). The sphere packing problem asks for the maximal value of $\Delta(\mathcal{P})$ and for which packing it is attained. Until 2017 the answer was only known for dimensions 1, 2 (see [16]), and 3. In 1611 Kepler conjectured that no sphere packing in $\mathbb{R}^{3}$ has density greater than $\pi \sqrt{2} / 6$ which is the density of the face-centered cubic lattice and the hexagonal close packing. The proof of the Kepler conjecture by Hales [19] was a major achievement and an endpoint of a long development (for a historical exposition see [1]).

The solution of the sphere packing problem in dimension 8 in March 2016 by Viazovska 32 and soon after in dimension 24 by Cohn, Kumar, Miller, Radchenko, and Viazovska [11] brought an enormous breakthrough in the application of linear programming techniques. For a comprehensive overview of the proof and more background information we refer to [8,13].

The proof was based on earlier work by Cohn and Elkies [7, 9], who provided the underlying linear programming technique. More precisely, the following result was proved there.

Theorem 1.1 (Theorem 3.2 in [9]). Suppose $f: \mathbb{R}^{d} \rightarrow \mathbb{R}$ is an admissible function satisfying the following three conditions for some $r>0$ :

\footnotetext{
Date: June 9, 2020.

*This author is supported by the Austrian Science Fund FWF project F5503 part of the Special Research Program (SFB) "Quasi-Monte Carlo Methods: Theory and Applications".

$\dagger$ The research of these authors was supported, in part, by the U. S. National Science Foundation under grant DMS-1516400.
} 
(1) $f(\mathbf{0})=\widehat{f}(\mathbf{0})>0$,

(2) $f(\mathbf{x}) \leq 0$ for $\|\mathbf{x}\| \geq r$

(3) $\widehat{f}(\mathbf{t}) \geq 0$ for all $\mathbf{t} \in \mathbb{R}^{d}$.

Then the density of sphere packings in $\mathbb{R}^{d}$ is bounded above by $(r / 2)^{d} B_{d}$, where $B_{d}$ denotes the volume of the d-dimensional unit ball.

A function $f$ is admissible, if there exists a constant $\delta>0$ such that $|f(\mathbf{x})|$ and $|\widehat{f}(\mathbf{x})|$ are bounded above by a constant times $(1+\|\mathbf{x}\|)^{-d-\delta}$. The admissibility condition implies the validity the Poisson summation formula for $f$ which plays a central role in the proof of the above result and further shows that the bound is attained for the lattice packing

$$
\mathcal{P}=\bigcup_{\mathbf{x} \in \Lambda} B\left(\mathbf{x}, \frac{r}{2}\right)
$$

for a lattice $\Lambda$, if and only if $f(\mathbf{x})=0$ for all $\mathbf{x} \in \Lambda \backslash\{\mathbf{0}\}$ and $\widehat{f}(\mathbf{t})=0$ for all $\mathbf{t} \in \Lambda^{*} \backslash\{\mathbf{0}\}$, where $\Lambda^{*}$ denotes the dual lattice of $\Lambda$, and $r$ is the minimal distance of $\Lambda$.

Of course, Schwartz functions are admissible. It is an important feature of the space of real valued radial Schwartz functions that every element $f$ can be written as $f=f_{+}+f_{-}$, where $\widehat{f}_{+}=f_{+}$and $\widehat{f}_{-}=-f_{-}$ are eigenfunctions of the Fourier transform. This is one of the key ingredients in the construction of functions $f$ satisfying the assumptions of the theorem.

The functions constructed in [11,32 were the first examples of Fourier eigenfunctions with prescribed double zeros at the distances of a lattice. The construction was based on Laplace transforms of certain weakly holomorphic modular forms and quasimodular forms. The aim of the present paper is to provide a unifying view on the modular forms behind these constructions, to construct the Fourier eigenfunctions for all dimensions divisible by 4 , and to show that the underlying modular and quasimodular forms are uniquely determined by the requirements that their transform should be an eigenfunction of the Fourier transform belonging to the Schwartz class.

Bourgain, Clozel, and Kahane [3] studied an uncertainty principle for the last sign change of even functions and their Fourier transforms on $\mathbb{R}$. More precisely, for dimension $d \geq 1$ let $\mathcal{A}_{+}(d)$ denote the set of functions $f: \mathbb{R}^{d} \rightarrow \mathbb{R}$ satisfying

(1) $f, \widehat{f} \in L^{1}\left(\mathbb{R}^{d}\right)$ and $\widehat{f}$ real valued, thus $f$ is even

(2) $f$ is eventually non-negative, while $\widehat{f}(\mathbf{0}) \leq 0$

(3) $\widehat{f}$ is eventually non-negative, while $f(\mathbf{0}) \leq 0$.

Similarly, denote by $\mathcal{A}_{-}(d)$ denote the set of functions $f: \mathbb{R}^{d} \rightarrow \mathbb{R}$ satisfying

(1) $f, \widehat{f} \in L^{1}\left(\mathbb{R}^{d}\right)$ and $\widehat{f}$ real valued, thus $f$ is even

(2) $f$ is eventually non-negative, while $\widehat{f}(\mathbf{0}) \leq 0$ 
(3) $\widehat{f}$ is eventually non-positive, while $f(\mathbf{0}) \geq 0$.

For such functions define

$$
r(f)=\inf \{R \geq 0 \mid f(\mathbf{x}) \text { has the same sign for }\|\mathbf{x}\| \geq R\}
$$

and set

$$
\begin{aligned}
& A_{+}(d)=\inf _{f \in \mathcal{A}_{+}(d)} \sqrt{r(f) r(\widehat{f})} \\
& A_{-}(d)=\inf _{f \in \mathcal{A}_{-}(d)} \sqrt{r(f) r(\widehat{f})} .
\end{aligned}
$$

This question was originally motivated by the study of zeta functions of number fields which have a real zero between 0 and 1 .

Gonçalves, Oliveira e Silva, and Steinerberger [17] studied the problem further and proved that the extremal functions for the above properties are eigenfunctions of the Fourier transform. Cohn and Gonçalves [10] used a construction similar to the ones in [11,32 to provide the optimal function for the above uncertainty principle in dimension 12 . They found $A_{+}(12)=\sqrt{2}$. Exact values of $A_{+}(d)$ and $A_{-}(d)$ are known only for very few dimensions (see [10]).

A point configuration $\mathcal{C} \subset \mathbb{R}^{d}$ is said to have density $\rho$, if

$$
\rho=\lim _{r \rightarrow \infty} \frac{\#(\mathcal{C} \cap B(\mathbf{0}, r))}{\operatorname{vol}_{d}(B(\mathbf{0}, r))},
$$

meaning that $\mathcal{C}$ contains $\rho$ points per unit volume. For a completely monotone function $p:(0, \infty) \rightarrow \mathbb{R}$ the $p$-energy of $\mathcal{C}$ is given by

$$
E_{p}(\mathcal{C})=\liminf _{r \rightarrow \infty} \frac{1}{\#(\mathcal{C} \cap B(\mathbf{0}, r))} \sum_{\substack{\mathbf{x}, \mathbf{y} \in \mathcal{C} \cap B(\mathbf{0}, r) \\ \mathbf{x} \neq \mathbf{y}}} p(\|\mathbf{x}-\mathbf{y}\|),
$$

which can be viewed as a thermodynamic limit of the sum of all mutual $p$-interactions of distinct points in $\mathcal{C}$. A configuration $\mathcal{C}$ of density $\rho$ is called universally optimal, if it minimises $E_{p}(\cdot)$ amongst all configurations of density $\rho$ and for all completely monotone functions $p$ simultaneously. Such configurations seem to exist only for special values of the dimension; only very few examples are known.

Radchenko and Viazovska [27] proved a remarkable interpolation theorem for functions on the real line, with prescribed values of $f$ and $\widehat{f}$ in the points $\pm \sqrt{n}\left(n \in \mathbb{N}_{0}\right)$. This idea was taken further by Cohn, Kumar, Miller, Radchenko, and Viazovska [12 in their proof of universal optimality of the $E_{8}$ and Leech lattices in respective dimensions 8 and 24. The main ingredient of their proof is an interpolation formula for radial Schwartz functions in these dimensions, which allows to interpolate values and first derivatives of $f$ and $\hat{f}$ in the points $\sqrt{2 n}$ $(n \in \mathbb{N})$.

As we were completing this manuscript we became aware of the work by Rolen and Wagner [28], who studied similar questions for dimensions 
divisible by 8 . They were focused on applications for proving packing bounds in these dimensions. These bounds turn out to be asymptotically weaker than the bounds known from work of Kabatjanskil and Levenšteĭn [22]. Our paper gives more explicit results especially for the underlying modular and quasimodular functions, in particular we find recurrence relations defining these functions.

In this paper the dimension $d$ will always be a multiple of 4 . It is organised as follows. In Section 2 we provide a general study of functions of the form

$$
U(s)=4 i \sin \left(\frac{\pi}{2} s\right)^{2} \int_{0}^{i \infty} \psi(z) e^{i \pi s z} d z
$$

for a class of functions $\psi$. Notice that the integral can be viewed as a Laplace transform after replacing $z=i t$ for $t>0$. We study the analytic continuation of such functions, which is already given in Viazovska's work [32. In Proposition 2.4 we formulate conditions on $\psi$ so that the function $U\left(\|\mathbf{x}\|^{2}\right)$ is an eigenfunction of the Fourier transform. These conditions turn out to be functional equations for $\psi$ and conditions on the asymptotic behaviour of $\psi(z)$ for $z \rightarrow 0$ and $z \rightarrow i \infty$. Our main aim is to find the function $\psi$ so that the last sign change of $U$ is as small as possible. This is motivated by the choice of functions in [11,32], as well as by the uncertainty principle [3] mentioned above.

In Section 3 we study the set of solutions of the functional equations given in Section 2 for the case of the eigenvalue $(-1)^{\frac{d}{4}}$. We show that the solutions are weakly holomorphic quasimodular forms of weight $4-\frac{d}{2}$ and depth 2. In order for the function $U\left(\|\mathbf{x}\|^{2}\right)$ to have the desired properties, we find conditions on these forms and show that these are uniquely satisfied.

In Section 4 the solutions of the functional equations from Section 2 for the case of the eigenvalue $(-1)^{\frac{d}{4}+1}$ are investigated. These turn out to be weakly holomorphic modular forms of weight $2-\frac{d}{2}$ for $\Gamma(2)$, a principal congruence subgroup of $\operatorname{SL}(2, \mathbb{Z})$. Again we characterise the functions $\psi$ that yield the desired properties for the function $U\left(\|\mathbf{x}\|^{2}\right)$.

In Section 5 we find differential equations satisfied by the forms obtained in Sections 3 and 4 and characterise them as certain solutions. Differential equations turn out to be a convenient method to control vanishing orders of quasimodular forms. As a byproduct we find linear recurrences for the forms.

In Section 6 we prove that all but possibly finitely many Fourier coefficients of the quasimodular forms obtained in Section 3 are positive.

In Section 7 we discuss the modular and quasimodular forms obtained in Sections 3 and 4 for several small dimensions, where the corresponding Fourier eigenfunction exhibits remarkable behaviour. These cases of course include dimensions 8, 12, and 24 . 
In Appendix $\mathrm{A}$ we provide some basic information on modular functions and forms, as well as quasimodular forms and derivatives of modular forms, that are needed for Sections 3 and 4 .

Notation. Throughout this paper we will use notations that are common in the context of modular forms. Especially, we denote the two generators of the modular group $\Gamma=\operatorname{PSL}(2, \mathbb{Z})$ by

$$
\begin{aligned}
& S: z \mapsto-\frac{1}{z} \\
& T: z \mapsto z+1 .
\end{aligned}
$$

Furthermore, we denote $q=e^{2 \pi i z}$, the nome, and use a slightly modified notation for derivatives

$$
f^{\prime}=\frac{1}{2 \pi i} \frac{d f}{d z}=q \frac{d f}{d q}
$$

We will freely switch between dependence on $z$ and $q$. The Landau symbol $\mathcal{O}\left(q^{\ell}\right)$ is always understood for $z \rightarrow i \infty$ which is $q \rightarrow 0$.

In this paper we use the following notation for the Fourier transform of a function $f \in L^{1}\left(\mathbb{R}^{d}\right)$ :

$$
\mathcal{F}(f)(\mathbf{t})=\widehat{f}(\mathbf{t}):=\int_{\mathbb{R}^{d}} f(\mathbf{x}) e^{-2 \pi i\langle\mathbf{x}, \mathbf{t}\rangle} d x_{1} \cdots d x_{d},
$$

where $\langle\mathbf{x}, \mathbf{t}\rangle$ denotes the standard scalar product in $\mathbb{R}^{d}$. With this setting we have

$$
\mathcal{F}\left(e^{\pi i z\|\mathbf{x}\|^{2}}\right)=(-1)^{d / 4} z^{-\frac{d}{2}} e^{\pi i S z\|\mathbf{t}\|^{2}}
$$

for the Fourier transform of a Gaussian for $\Im z>0$. Here and throughout this paper $\|\mathbf{x}\|^{2}=\langle\mathbf{x}, \mathbf{x}\rangle$ denotes the euclidean norm.

\section{LAPLACE TRANSFORMS AND FOURIER EIGENFUNCTIONS}

In this section we give a general study of functions given in the form (1.2). This representation is one of the key ingredient of Viazovska's construction of eigenfunctions of the Fourier transform. We analyse these functions in some detail and provide their analytic continuation to a right half-plane containing the imaginary axis. After these preparations we compute the Fourier transform of the function $U\left(\|\mathbf{x}\|^{2}\right)$ and use this to obtain necessary and sufficient conditions for this function to be an eigenfunction of the Fourier transform (Proposition 2.4). This is the starting point for the considerations in Sections 3 and 4 . We denote the non-negative imaginary axis by $i \mathbb{R}_{+}:=i(0, \infty)$ and let $L_{\text {loc }}^{1}\left(i \mathbb{R}_{+}\right)$ denote the complex valued functions that are absolutely integrable with respect to Lebesgue measure on any bounded interval $i(0, b]$. 
Proposition 2.1. Suppose $\psi \in L_{\mathrm{loc}}^{1}\left(i \mathbb{R}_{+}\right)$is such that for some $C>0$ and constants $a_{k}, b_{k} \in \mathbb{C}, k=0,1, \ldots, n$,

$$
\psi(z)=\sum_{k=0}^{n} a_{k} e^{-2 \pi i k z}-i z \sum_{k=0}^{n} b_{k} e^{-2 \pi i k z}+\mathcal{O}\left(e^{i C z}\right) \quad \text { as } z \rightarrow i \infty .
$$

For $\Re(s)>2 n$, let

$$
W(s):=-i \int_{0}^{i \infty} \psi(z) e^{i \pi s z} d z
$$

Then

$$
\begin{aligned}
& W(s)=\sum_{k=0}^{n}\left(\frac{a_{k}}{\pi(s-2 k)}+\frac{b_{k}}{\pi^{2}(s-2 k)^{2}}\right) \\
& -i \int_{0}^{i \infty}\left(\psi(z)-\left(\sum_{k=0}^{n} a_{k} e^{-2 \pi i k z}+z \sum_{k=0}^{n} b_{k} e^{-2 \pi i k z}\right)\right) e^{i \pi s z} d z .
\end{aligned}
$$

gives an analytic continuation of $W$ to the half-plane $\Re(s)>-C / \pi$.

Proof. Let $\widetilde{W}(s)$ be given by the right-hand side of (2.3). Then the local integrability of $\psi$ and the condition (2.1) imply that $\widetilde{W}(s)$ is a well-defined meromorphic function on the half plane $\Re(s)>-\frac{C}{\pi}$ with (at most) double poles at $s=2 k, k=0, \ldots, n$. For an integer $k$ and $\Re(s)>2 k$, elementary computations show

$$
-i \int_{0}^{i \infty} e^{-2 \pi i k z} e^{i \pi s z} d z=\frac{1}{\pi(s-2 k)}
$$

and

$$
-\int_{0}^{i \infty} z e^{-2 \pi i k z} e^{i \pi s z} d z=\frac{1}{\pi^{2}(s-2 k)^{2}}
$$

and hence that $\widetilde{W}(s)$ agrees with $W(s)$ for $\Re(s)>2 n$.

We next assume that $\psi$ is holomorphic on the upper half-plane.

Proposition 2.2. Let $\psi: \mathbb{H} \rightarrow \mathbb{C}$ be holomorphic on $\mathbb{H}$ and bounded on the angular region $R_{\alpha, \epsilon}:=\left\{r e^{i t}: 0<r<\epsilon, \alpha<t<\pi-\alpha\right\}$ for some $\epsilon>0$ and some $0<\alpha<\pi / 4$. Further suppose the restriction of $\psi$ to $i \mathbb{R}_{+}$and $W$ are as in Proposition 2.1 and for $\Re(s)>-C / \pi$ let $U(s)$ be defined by

$$
U(s):=-4 \sin \left(\frac{\pi}{2} s\right)^{2} W(s) .
$$




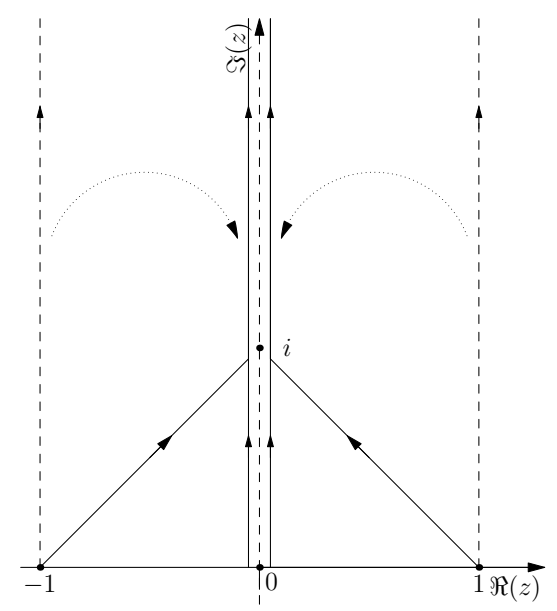

FiguRE 2.1. Deforming the contour of integration

Then $U(s)$ is holomorphic for $\Re(s)>-C / \pi$ and

$$
\begin{aligned}
& i U(s)=\int_{-1}^{i} \psi(T z) e^{i \pi s z} d z+\int_{1}^{i} \psi\left(T^{-1} z\right) e^{i \pi s z} d z \\
& -2 \int_{0}^{i} \psi(z) e^{i \pi s z} d z+\int_{i}^{i \infty}\left(\psi(T z)-2 \psi(z)+\psi\left(T^{-1} z\right)\right) e^{i \pi s z} d z
\end{aligned}
$$

where the integrals are along straight line segments joining the endpoints.

Proof. Starting from (2.2) we derive a second form of the analytic continuation of $-4 \sin \left(\frac{\pi}{2} s\right)^{2} W(s)$, which is more suitable for the proof and will also be used later. We write

$$
\begin{aligned}
i U(s) & =\int_{0}^{i \infty} \psi(z)\left(e^{i \pi s(z-1)}-2 e^{i \pi s z}+e^{i \pi s(z+1)}\right) d z \\
& =\int_{-1}^{-1+i \infty} \psi(T z) e^{i \pi s z} d z-2 \int_{0}^{i \infty} \psi(z) e^{i \pi s z} d z \\
& +\int_{1}^{1+i \infty} \psi\left(T^{-1} z\right) e^{i \pi s z} d z,
\end{aligned}
$$

which follows by expressing the sine in terms of the exponential, expanding the square and substituting in the integral. This expression is valid for $\Re(s)>2 n$. Since $\psi$ is holomorphic on $\mathbb{H}$, bounded on $R_{\alpha, \epsilon}$ and satisfies the growth condition (2.1), we may deform the contours of integration as follows: the path from -1 to $-1+i \infty$ is deformed into a straight line from -1 to $i$ and then along the imaginary axis from $i$ to $i \infty$; similarly, the contour from 1 to $1+i \infty$ is deformed into a straight line from 1 to $i$ and then again along the imaginary axis (see Figure 2.1). 
Collecting terms with matching paths of integration gives (2.5) valid for $\Re(s)>2 n$. Since the exponential terms in the asymptotic expansion (2.1) for $z \rightarrow i \infty$ cancel in the last integral, the new expression is also valid for $\Re(s)>-\frac{C}{\pi}$ providing an alternative form for expressing the analytic continuation of $U(s)$. The integrals are all absolutely and uniformly convergent for $\Re(s) \geq 0$.

Proposition 2.3. Let $\psi$ and $U$ be as in Proposition 2.2, and let F : $\mathbb{R}^{d} \rightarrow \mathbb{C}$ be defined by

$$
F(\mathbf{x}):=U\left(\|\mathbf{x}\|^{2}\right), \quad\left(\mathbf{x} \in \mathbb{R}^{d}\right) .
$$

If, in addition, $\psi$ satisfies

$$
\psi(z)=\mathcal{O}\left(e^{i C S z}\right) \quad \text { as } z \rightarrow 0 \quad \text { non-tangentially in } \mathbb{H},
$$

then $F$ is a Schwartz function and can be written in the form

$$
\begin{aligned}
& F(\mathbf{x})=-i\left[\int_{-1}^{i} \psi(T z) e^{i \pi\|\mathbf{x}\|^{2} z} d z+\int_{1}^{i} \psi\left(T^{-1} z\right) e^{i \pi\|\mathbf{x}\|^{2} z} d z\right. \\
& \left.\quad-2 \int_{0}^{i} \psi(z) e^{i \pi\|\mathbf{x}\|^{2} z} d z+\int_{i}^{i \infty}\left(\psi(T z)-2 \psi(z)+\psi\left(T^{-1} z\right)\right) e^{i \pi\|\mathbf{x}\|^{2} z} d z\right] .
\end{aligned}
$$

Consequently, the Fourier transform of $F$ is given by

$$
\begin{aligned}
& \hat{F}(\mathbf{t})=-i(-1)^{d / 4}\left[\int_{-1}^{i} \psi\left(T^{-1} S z\right) e^{i \pi\|\mathbf{t}\|^{2} z} z^{d / 2-2} d z\right. \\
& +2 \int_{i}^{i \infty} \psi(S z) e^{i \pi\|\mathbf{t}\|^{2} z} z^{d / 2-2} d z+\int_{1}^{i} \psi(T S z) e^{i \pi\|\mathbf{t}\|^{2} z} z^{d / 2-2} d z \\
& \left.-\int_{0}^{i}\left(\psi\left(T^{-1} S z\right)-2 \psi(S z)+\psi(T S z)\right) e^{i \pi\|\mathbf{t}\|^{2} z} z^{d / 2-2} d z\right] .
\end{aligned}
$$

Proof. The representation (2.8) follows immediately from the definition (2.6) and the relation (2.5) of Proposition 2.2. Condition (2.7) implies that $\psi$ vanishes to arbitrary order at $z=0$. Hence, using (2.2) it follows using well known properties of the Laplace transform (see [33]) that $F$ and its derivatives all decay faster than any negative power of $\|\mathbf{x}\|$. Since $U$ is analytic, it follows that $F$ is a Schwartz function.

Thus we can compute the Fourier transform of $F$ by Fubini's theorem

$$
\begin{aligned}
\widehat{F}(\mathbf{t})= & -i\left[\int_{-1}^{i} \psi(T z) e^{i \pi\|\mathbf{t}\|^{2} S z}(-i z)^{-\frac{d}{2}} d z\right. \\
& +\int_{1}^{i} \psi\left(T^{-1} z\right) e^{i \pi\|\mathbf{t}\|^{2} S z}(-i z)^{-\frac{d}{2}} d z-2 \int_{0}^{i} \psi(z) e^{i \pi\|\mathbf{t}\|^{2} S z}(-i z)^{-\frac{d}{2}} d z \\
& \left.+\int_{i}^{i \infty}\left(\psi(T z)-2 \psi(z)+\psi\left(T^{-1} z\right)\right) e^{i \pi\|\mathbf{t}\|^{2} S z}(-i z)^{-\frac{d}{2}} d z\right]
\end{aligned}
$$

Substituting $S z$ in this expression and collecting signs gives (2.9)). 
Proposition 2.4. Let $\psi$ and $F$ be as in Proposition 2.3 and $\varepsilon \in$ $\{-1,1\}$. Then $\hat{F}=\varepsilon(-1)^{\frac{d}{4}} F$ if and only if

$$
\begin{aligned}
z^{\frac{d}{2}-2} \psi\left(T^{-1} S z\right) & =\varepsilon \psi(T z) \\
2 z^{\frac{d}{2}-2} \psi(S z) & =\varepsilon\left(\psi(T z)-2 \psi(z)+\psi\left(T^{-1} z\right)\right),
\end{aligned}
$$

for all $z \in \mathbb{H}$.

Proof. We consider the auxiliary function

$$
H(s)=\sum_{\ell=1}^{4} I_{\ell}(s),
$$

where

$$
\begin{aligned}
& \left.I_{1}(s)=\int_{i}^{i \infty}\left(\psi(T z)-2 \psi(z)+\psi\left(T^{-1} z\right)-2 \varepsilon z^{d / 2-2} \psi(S z)\right)\right) e^{i \pi s z} d z \\
& I_{2}(s)=\int_{0}^{i}\left(\varepsilon\left(\psi(T S z)-2 \psi(S z)+\psi\left(T^{-1} S z\right)\right) z^{d / 2-2}-2 \psi(z)\right) e^{i \pi s z} d z \\
& I_{3}(s)=\int_{-1}^{i}\left(\psi(T z)-\varepsilon \psi\left(T^{-1} S z\right) z^{d / 2-2}\right) e^{i \pi s z} d z \\
& I_{4}(s)=\int_{1}^{i}\left(\psi\left(T^{-1} z\right)-\varepsilon \psi(T S z) z^{d / 2-2}\right) e^{i \pi s z} d z
\end{aligned}
$$

Then $H\left(\|\mathbf{x}\|^{2}\right)=F(\mathbf{x})-\varepsilon(-1)^{\frac{d}{4}} \widehat{F}(\mathbf{x})$. If $F$ is an eigenfunction for the Fourier transform with eigenvalue $\varepsilon(-1)^{\frac{d}{4}}$, then $H(s)$ vanishes on the positive real axis and thus for all $s \in \mathbb{C}$.

Now the functions $I_{2}, I_{3}, I_{4}$ are entire functions of exponential type $\pi$ (see [2]). Thus for $H(s)$ to vanish identically, $I_{1}(s)$ also has to be of exponential type. By the Paley-Wiener theorem (see [26]) this implies that the integrand defining $I_{1}$ has to have compact support. Since the integrand is analytic it has to vanish identically giving

$$
2 z^{\frac{d}{2}-2} \psi(S z)=\varepsilon\left(\psi(T z)-2 \psi(z)+\psi\left(T^{-1} z\right)\right) .
$$

Since the integrand in the definition of $I_{2}$ equals the integrand for $I_{1}$ after a substitution $z \mapsto S z$, also $I_{2}$ vanishes identically.

Now it remains $H(s)=I_{3}(s)+I_{4}(s)=0$. We observe that

$$
\begin{aligned}
& \lim _{t \rightarrow-\infty} I_{3}(t)=\infty, \quad \lim _{t \rightarrow \infty} I_{3}(t)=0 \\
& \lim _{t \rightarrow+\infty} I_{4}(t)=\infty, \quad \lim _{t \rightarrow-\infty} I_{4}(t)=0 .
\end{aligned}
$$

Thus $H(s)=0$ can only hold, if $I_{3}$ and $I_{4}$ vanish identically. Again both integrals $I_{3}$ and $I_{4}$ can be rewritten as Laplace transforms after a 
change of variables. It follows that

$$
\begin{aligned}
z^{\frac{d}{2}-2} \psi\left(T^{-1} S z\right) & =\varepsilon \psi(T z) \\
z^{\frac{d}{2}-2} \psi(T S z) & =\varepsilon \psi\left(T^{-1} z\right) .
\end{aligned}
$$

It is immediate that (2.16) and (2.17) are equivalent by substituting $z \mapsto S z$.

\section{The $(-1)^{\frac{d}{4}}$ EIGENFUNCTION}

In this section we first prove that the function $\psi$ in (2.2) has to be a quasimodular form in order to make $F$ given by (2.8) an eigenfunction of the Fourier transform for the eigenvalue $(-1)^{\frac{d}{4}}$. The main ingredient for this fact is Proposition 3.1 below, which is essentially equivalent to [12, Proposition 4.7] except for different growth conditions on the function $\psi$. We developed this result independently, and keep the proof in order to make the paper self-contained. In Section 3.1 we then use dimension arguments for the underlying spaces of quasimodular forms to achieve the existence of such forms that are useful for the construction of eigenfunctions with certain extremal properties.

Proposition 3.1. Let $\psi$ be as in Proposition 2.2. Then the corresponding function $F$ given by (2.6) is an eigenfunction for the Fourier transform with eigenvalue $(-1)^{\frac{d}{4}}$, if and only if $z^{\frac{d}{2}-2} \psi(S z)$ is a weakly holomorphic quasimodular form of weight $4-\frac{d}{2}$ and depth 2. More precisely, there are weakly holomorphic modular forms $\psi_{1}, \psi_{2}, \psi_{3}$ of respective weights $4-\frac{d}{2}, 2-\frac{d}{2}$, and $-\frac{d}{2}$ such that

$$
z^{\frac{d}{2}-2} \psi(S z)=\psi_{1}(z)-2 E_{2}(z) \psi_{2}(z)+E_{2}(z)^{2} \psi_{3}(z) .
$$

This gives

$$
\begin{aligned}
\psi(z) & =z^{2}\left(\psi_{1}(z)-2 E_{2}(z) \psi_{2}(z)+E_{2}(z)^{2} \psi_{3}(z)\right) \\
& +z \frac{12 i}{\pi}\left(\psi_{2}(z)-E_{2}(z) \psi_{3}(z)\right)-\frac{36}{\pi^{2}} \psi_{3}(z) .
\end{aligned}
$$

Furthermore, $\psi_{1}, \psi_{2}$, and $\psi_{3}$ have to satisfy

$$
\psi_{1}(z)-2 E_{2}(z) \psi_{2}(z)+E_{2}(z)^{2} \psi_{3}(z)=\mathcal{O}\left(e^{2 \pi i z}\right)
$$

for $z \rightarrow i \infty$ in order to fulfil (2.1) and (2.7).

Proof. By Proposition 2.4 a function $F$ given in the form (2.4) is an eigenfunction of the Fourier transform for the eigenvalue $(-1)^{\frac{d}{4}}$ (this is $\varepsilon=1$ ) if and only if (2.10) and (2.11) hold. From (2.10) we obtain

$$
\psi(z)=(z+1)^{\frac{d}{2}-2} \psi(T S T z)
$$

and then

$$
(z+1)^{\frac{d}{2}-2} \psi(S T z)=z^{\frac{d}{2}-2} \psi(T S T S T z)=z^{\frac{d}{2}-2} \psi(S z),
$$


where we have used that $(T S)^{3}=\mathrm{id}$. Thus the function

$$
\phi(z)=z^{\frac{d}{2}-2} \psi(S z)
$$

is periodic with period 1 .

Now we write (2.11) as

$$
\psi(T z)-2 \psi(z)+\psi\left(T^{-1} z\right)=2 \phi(z)
$$

and set

$$
f(z)=\psi(T z)-\psi(z)-(2 z+1) \phi(z) .
$$

Then we have

$$
\begin{aligned}
& f(z)-f\left(T^{-1} z\right) \\
& \quad=\psi(T z)-2 \psi(z)+\psi\left(T^{-1} z\right)-(2 z+1) \phi(z)+(2 z-1) \phi\left(T^{-1} z\right) .
\end{aligned}
$$

Using the periodicity of $\phi$ and (3.4) gives the periodicity of $f$. Now we set

$$
g(z)=\psi(z)-z^{2} \phi(z)-z f(z)
$$

We compute

$g(T z)-g(z)=\psi(T z)-\psi(z)-\left((z+1)^{2}-z^{2}\right) \phi(z)-((z+1)-z) f(z)=0$,

where we have used the periodicity of $\phi$ and $f$ as well as the definition of $f$. This shows that also $g$ is periodic.

Thus $\psi$ satisfies the relation

$$
\psi(z)=z^{\frac{d}{2}} \psi(S z)+z f(z)+g(z)
$$

for two (yet unknown) periodic functions $f$ and $g$. We now use definitions (3.5) and (3.6) to express $g$ in terms of $\psi$

$$
g(z)=(z+1) \psi(z)-z \psi(T z)+z(z+1) z^{\frac{d}{2}-2} \psi(S z) .
$$

Substituting $S T z$ and multiplying through the denominator yields

$$
\begin{aligned}
(z+1)^{\frac{d}{2}} g(S T z) & =z(z+1)(z+1)^{\frac{d}{2}-2} \psi(S T z) \\
& +(z+1)(z+1)^{\frac{d}{2}-2} \psi\left(S T^{-1} S z\right)-z \psi(T z),
\end{aligned}
$$

where we have used $T S T=S T^{-1} S$. We have already established the periodicity of $\phi(z)=z^{\frac{d}{2}-2} \psi(S z)$. This allows to replace the first and the second term to yield

$$
g(S T z)=(z+1) z^{\frac{d}{2}-1} \psi(S z)+(z+1) \psi(z)-z \psi(T z)=g(z) .
$$

Using the already established periodicity of $g$ this gives

$$
z^{\frac{d}{2}} g(S z)=g(z)
$$

together with the growth condition (2.1) this shows that $g$ is a weakly holomorphic modular form of weight $-\frac{d}{2}$. 
Applying $z \mapsto S z$ to (3.7) and adding this to (3.7) (divided by $z^{\frac{d}{2}}$ ) yields

$$
z^{\frac{d}{2}-2} f(S z)=f(z)+\frac{2}{z} g(z)
$$

$f$ is a weakly holomorphic quasimodular form of weight $2-\frac{d}{2}$ and depth 1 (again using (2.1)).

We set

$$
h(z)=f(z)-\frac{\pi i}{3} E_{2}(z) g(z)
$$

and use $z^{-2} E_{2}(z)=E_{2}(z)-\frac{6 i}{\pi z}$ to obtain

$$
z^{\frac{d}{2}-2} h(S z)=h(z)
$$

which together with the obvious periodicity and the growth condition (2.1) yields that $h$ is a weakly holomorphic modular form of weight $2-\frac{d}{2}$. Inserting this into (3.7) gives the quasimodularity of $z^{\frac{d}{2}-2} \psi(S z)$ with weight $4-\frac{d}{2}$ and depth 2 . By the structure theorem of quasimodular forms (see [25, 29, 36]), $z^{\frac{d}{2}-2} \psi(S z)$ can then be written as (3.1), where we have set

$$
\begin{aligned}
& \psi_{1}(z)=z^{\frac{d}{2}-2} \psi(S z)-E_{2}(z) h(z)-E_{2}(z)^{2} g(z) \\
& \psi_{2}(z)=-\frac{\pi i}{12} h(z) \\
& \psi_{3}(z)=-\frac{\pi^{2}}{36} g(z) .
\end{aligned}
$$

In order to satisfy condition (2.1), the term multiplied by $z^{2}$ in (3.2) has to tend to 0 for $z \rightarrow i \infty$, which gives (3.3). By (3.1) this implies that (2.1) and (2.7) are satisfied for any $0<C<2 \pi$.

3.1. Determining $\psi$. In a next step we want to determine $\psi$ (or equivalently $\psi_{1}, \psi_{2}, \psi_{3}$ ) to satisfy (3.3). Since $\psi_{1}, \psi_{2}$, and $\psi_{3}$ are weakly holomorphic modular forms of respective weights $4-\frac{d}{2}, 2-\frac{d}{2}$, and $-\frac{d}{2}$, we express these forms as

$$
\begin{aligned}
& \psi_{1}=\frac{1}{\Delta^{\ell}} \omega_{k+2} P_{n}^{(k)}(j) \\
& \psi_{2}=\frac{1}{\Delta^{\ell}} \omega_{k+1} Q_{n}^{(k)}(j) \\
& \psi_{3}=\frac{1}{\Delta^{\ell}} \omega_{k} R_{n}^{(k)}(j),
\end{aligned}
$$

for $\ell \in \mathbb{N}$ chosen so that $\psi_{m} \Delta^{\ell}(m=1,2,3)$ are weakly holomorphic modular forms of positive weight; $P_{n}^{(k)}, Q_{n}^{(k)}$, and $R_{n}^{(k)}$ are polynomials, which have to be determined. The parameter $n$ is an ordering parameter related to the degrees of these polynomials. It will be determined by (3.15) in the course of the following discussion. 
The minimal possible choice of $\ell$ is then

$$
\ell=\left\lceil\frac{d}{24}\right\rceil \text {. }
$$

Furthermore, we set

$$
k=6 \ell-\frac{d}{4},
$$

which gives $0 \leq k \leq 5$. The forms $\omega_{m}$ in (3.12) are modular forms of weight $2 m(m=0, \ldots, 7)$, which are given in Table 3.1, these forms are uniquely determined by the requirement to be holomorphic, or to have a pole of minimal order at $i \infty$. The parameter $n$ refers to the order of the pole of $\omega_{k+2} P_{n}^{(k)}(j), \omega_{k+1} Q_{n}^{(k)}(j)$, or $\omega_{k} R_{n}^{(k)}(j)$. Notice that for $m=1$ the form $\omega_{m}$ has a simple pole at $i \infty$, whereas for $m=6,7$ it has a simple zero there. This affects the possible degrees of the polynomials $P_{n}^{(k)}, Q_{n}^{(k)}$, and $R_{n}^{(k)}$, see Table 3.2. This table also gives the dimension of the space $\mathcal{Q}_{n}^{(2 k+2)}$ of weakly holomorphic quasimodular forms of weight $2 k+2$ and depth 2 , which have a pole of order at most $n$ at $i \infty$. The table also gives the definition of the quantity $a(k)$, which will be needed in the sequel.

\begin{tabular}{|l|c|}
\hline$m$ & $\omega_{m}$ \\
\hline 0 & 1 \\
\hline 1 & $-j^{\prime}=\frac{E_{4}^{2} E_{6}}{\Delta}=\frac{E_{6}}{E_{4}} j$ \\
\hline 2 & $E_{4}$ \\
\hline 3 & $E_{6}$ \\
\hline 4 & $E_{4}^{2}$ \\
\hline 5 & $E_{4} E_{6}$ \\
\hline 6 & $\Delta=\Delta \omega_{0}$ \\
\hline 7 & $E_{4}^{2} E_{6}=\Delta \omega_{1}$ \\
\hline
\end{tabular}

TABLE 3.1. The choices of $\omega_{m}$

In light of (3.2) and the asymptotic behaviour of $\psi(2.1)$ used in Proposition 2.1 we require that the polar order of $\psi_{2}(z)-E_{2}(z) \psi_{3}(z)$ (the term multiplied by $z$ in (3.2)) is 1 less than the polar order of $\psi_{3}(z)$. This ensures by (2.3) that the largest real second order pole of $W(s)$ is 2 less than the largest real first order pole. Notice that condition (3.3) ensures that $W(s)$ has no third order poles in the right half plane. Together this ensures that the polar order of $\psi$ at $i \infty$ corresponds to the desired sign change of the function $F$ given by (2.6).

In order to achieve the behaviour of $\psi$ described in the last paragraph, we use the degrees of freedom given by $\operatorname{dim} \mathcal{Q}_{n}^{(2 k+2)}$ to first ensure 


\begin{tabular}{|c|c|c|c|c|c|}
\hline$k$ & $\operatorname{deg} P_{n}^{(k)}$ & $\operatorname{deg} Q_{n}^{(k)}$ & $\operatorname{deg} R_{n}^{(k)}$ & $\operatorname{dim} \mathcal{Q}_{n}^{(2 k+2)}$ & $a(k)$ \\
\hline 0 & $n$ & $n-1$ & $n$ & $3 n+2$ & 1 \\
\hline 1 & $n$ & $n$ & $n-1$ & $3 n+2$ & 1 \\
\hline 2 & $n$ & $n$ & $n$ & $3 n+3$ & 2 \\
\hline 3 & $n$ & $n$ & $n$ & $3 n+3$ & 2 \\
\hline 4 & $n+1$ & $n$ & $n$ & $3 n+4$ & 3 \\
\hline 5 & $n$ & $n+1$ & $n$ & $3 n+4$ & 3 \\
\hline
\end{tabular}

TABLE 3.2. Degrees of the polynomials $P_{n}^{(k)}, Q_{n}^{(k)}$, and $R_{n}^{(k)}$

that

$$
\omega_{k+1} Q_{n}^{(k)}(j)-E_{2} \omega_{k} R_{n}^{(k)}(j)=\mathcal{O}\left(q^{-n+1}\right)
$$

and second to eliminate as many Laurent series coefficients of

$$
\omega_{k+2} P_{n}^{(k)}(j)-2 E_{2} \omega_{k+1} Q_{n}^{(k)}(j)+E_{2}^{2} \omega_{k} R_{n}^{(k)}(j)
$$

as possible. By solving the according linear equations we can achieve

$$
\omega_{k+2} P_{n}^{(k)}(j)-2 E_{2} \omega_{k+1} Q_{n}^{(k)}(j)+E_{2}^{2} \omega_{k} R_{n}^{(k)}(j)=\mathcal{O}\left(q^{2 n+a(k)-1}\right) .
$$

In order for $\psi$ to satisfy (3.3) we have to choose $n$ so that

$$
2 n+a(k)-1>\ell
$$

the minimal possible choice for $n$ is then

$$
n=\left\lceil\frac{\ell-a(k)+2}{2}\right\rceil \text {. }
$$

Condition (3.13) ensures that there is a sign change of $F(\mathbf{x})$ at $\|\mathbf{x}\|^{2}=2 n+2 \ell$ and $F(\mathbf{x}) \neq 0$ for $\|\mathbf{x}\|^{2}=2 n+2 \ell-2$. Expressing $\ell, k$, and $n$ in terms of $d$ yields $2 n+2 \ell=2\left\lfloor\frac{d+4}{16}\right\rfloor+2$.

Summing up, we have proved most of the following theorem. For the sake of simplicity, we abuse notation by writing $f(\mathbf{x})=f(\|\mathbf{x}\|)$, whenever $f$ is a radial function and the context is clear.

Theorem 3.2. For $d \equiv 0(\bmod 4)$ set $n_{+}=\left\lfloor\frac{d+4}{16}\right\rfloor+1$. Then there exists a radial Schwartz function $F_{+}: \mathbb{R}^{d} \rightarrow \mathbb{R}$ satisfying

$$
\begin{aligned}
F_{+}(\mathbf{x}) & =(-1)^{\frac{d}{4}} \widehat{F}_{+}(\mathbf{x}) \quad \text { for all } \mathbf{x} \in \mathbb{R}^{d} \\
F_{+}\left(\sqrt{2 n_{+}}\right) & =0 \quad \text { and } F_{+}^{\prime}\left(\sqrt{2 n_{+}}\right) \neq 0 \\
F_{+}(\sqrt{2 m}) & =F_{+}^{\prime}(\sqrt{2 m})=0 \quad \text { for } m>n_{+}, \quad m \in \mathbb{N} .
\end{aligned}
$$

If Conjecture 1 stated below holds, then $\sqrt{2 n_{+}}$is the last sign change of the function $F_{+}$. This is the case for all dimensions $\leq 312$ by Remark 6.3 stated below. 


\begin{tabular}{|c|c|c|c|c|c|c|}
\hline & \multicolumn{2}{|c|}{$d$} & $n$ & $P_{n}^{(k)}(w)$ & $Q_{n}^{(k)}(w)$ & $R_{n}^{(k)}(w)$ \\
\hline \multirow[t]{2}{*}{$k=0$} & & 24 & 1 & $w-3528$ & 1 & $w+1800$ \\
\hline & 48 & 72 & 2 & $\begin{array}{l}175 w^{2}- \\
1840638 w \\
-475793136\end{array}$ & $175 w+497922$ & $\begin{array}{l}175 w^{2}+ \\
2534082 w \\
+111078000\end{array}$ \\
\hline \multirow[t]{2}{*}{$k=1$} & & 20 & 1 & $w-1008$ & $w-1368$ & 1 \\
\hline & 44 & 68 & 2 & $\begin{array}{l}25 w^{2}-167286 w \\
-10456992\end{array}$ & $\begin{array}{l}25 w^{2}-18966 w \\
-41044752\end{array}$ & $25 w+172554$ \\
\hline \multirow[t]{3}{*}{$k=2$} & & & 0 & 1 & 1 & 1 \\
\hline & 16 & 40 & 1 & $w-5628$ & $w+420$ & $w+4740$ \\
\hline & 64 & 88 & 2 & $\begin{array}{l}21 w^{2}-277373 w \\
-147949620\end{array}$ & $\begin{array}{l}21 w^{2}+104155 w \\
+2942940\end{array}$ & $\begin{array}{l}21 w^{2}+449395 w \\
+62398380\end{array}$ \\
\hline \multirow[t]{3}{*}{$k=3$} & & & 0 & 1 & 1 & 1 \\
\hline & 12 & 36 & 1 & $w-2548$ & $w-1588$ & $w+1100$ \\
\hline & 60 & 84 & 2 & $\begin{array}{l}7 w^{2}-63953 w \\
-13216476 \\
\end{array}$ & $\begin{array}{l}7 w^{2}+3079 w \\
-26138316\end{array}$ & $\begin{array}{l}7 w^{2}+82207 w \\
+2838660\end{array}$ \\
\hline \multirow[t]{2}{*}{$k=4$} & & 8 & 0 & $w-1728$ & 1 & 1 \\
\hline & 32 & 56 & 1 & $\begin{array}{l}5 w^{2}-39879 w- \\
3302208\end{array}$ & $5 w+6741$ & $5 w+44721$ \\
\hline \multirow[t]{2}{*}{$k=5$} & & 4 & 0 & 1 & $w-864$ & 1 \\
\hline & 28 & 52 & 1 & $w-4473$ & $\begin{array}{l}w^{2}-1413 w- \\
453600\end{array}$ & $w+3375$ \\
\hline
\end{tabular}

TABLE 3.3. The polynomials $P_{n}^{(k)}, Q_{n}^{(k)}$, and $R_{n}^{(k)}$; the dimensions in the left column are those covered by Remark 3.3 .

Proof. It only remains to show that $F_{+}^{\prime}\left(\sqrt{2 n_{+}}\right) \neq 0$. This follows from the discussion of the vanishing orders in Section 5.1, especially equation (5.5) and the explanation following. More precisely, (5.5) implies that $a_{n_{+}} \neq 0$ in (2.1), whereas (5.4) implies that $b_{n_{+}}=0$. This shows by (2.3) that $W$ has a simple pole at $s=2 n_{+}$, which gives a simple zero of $U$ at this point.

Remark 3.3. Notice that by (3.14) the order increases by 2 , if $n$ increases by 1 . Together with (3.15) this gives that for certain $d(\bmod 48)$ the order of vanishing of $\psi$ is one more than required. This means that for these values of the dimension there is one extra degree of freedom, that can be used for instance to require $F(\mathbf{0})=0$. This is the case for dimensions

$$
d \equiv\{0,12,16,28,32,44\} \quad(\bmod 48)
$$




\section{Eigenfunctions For eigenvalue $(-1)^{\frac{d}{4}+1}$}

In this section we consider eigenfunctions of the Fourier transform with eigenvalue $(-1)^{\frac{d}{4}+1}$ of the form (1.2). We show in Proposition 4.1 that, in this case, the function $\psi$ can be expressed in terms of weakly holomorphic modular forms for $\Gamma$ and $\Gamma(2)$. This proposition is essentially equivalent to [12, Proposition 4.8], except for different growth conditions in the assumptions. This result was found independently; we keep the proof in order to keep the presentation self-contained. We then explore explicit representations of these forms and show the existence of eigenfunctions satisfying similar extremal properties as in Section 3 .

In the following the function $\log \lambda$ ( $\lambda$ being the Hauptmodul for $\Gamma(2))$ will play an important role. Properties of this function are discussed in Appendix A.

Proposition 4.1. Let $\psi$ be as in Proposition 2.2. Then the corresponding function $F$ given by (2.6) is an eigenfunction of the Fourier transform with eigenvalue $(-1)^{\frac{d}{4}+1}$ if and only if there exists a weakly holomorphic modular form $f$ of weight $2-\frac{d}{2}$ for $\Gamma$ and $\omega$ a weakly holomorphic modular form of weight $2-\frac{d}{2}$ for $\Gamma(2)$ such that

$$
\begin{aligned}
& \psi(z)=f(z) \cdot \log \lambda(z)+\omega(z), \\
& \omega(z)=z^{\frac{d}{2}-2} \omega(S z)+\omega(T z),
\end{aligned}
$$

where $\log \lambda$ is defined in (A.18).

Proof. By Proposition 2.4 with $\epsilon=-1, F$ is an eigenfunction of the Fourier transform with eigenvalue $(-1)^{\frac{d}{4}+1}$ iff $\psi$ satisfies the two equations:

$$
\begin{aligned}
z^{\frac{d}{2}-2} \psi(T S z) & =-\psi\left(T^{-1} z\right) \\
2 z^{\frac{d}{2}-2} \psi(S z) & =-\left(\psi(T z)-2 \psi(z)+\psi\left(T^{-1} z\right)\right) .
\end{aligned}
$$

To solve these we first consider $H(z):=z^{\frac{d}{2}-2} \psi(S z)$ which by (4.3) gives

$$
\begin{aligned}
H(T z) & =(T z)^{\frac{d}{2}-2} \psi(S T z)=(T z)^{\frac{d}{2}-2} \psi\left(T^{-1} T S T z\right) \\
& =-(T z)^{\frac{d}{2}-2}(T S T z)^{\frac{d}{2}-2} \psi(T S T S T z) \\
& =-z^{\frac{d}{2}-2} \psi(S z)=-H(z)
\end{aligned}
$$

Where we used the property $(T S)^{3}=\mathrm{id}$ in the middle line. Iterating this property once gives that $H(z+2)=H(z)$ and unravelling this statement in terms of $\psi$ gives

$$
(2 z-1)^{\frac{d}{2}-2} \psi\left(S T^{2} S z\right)=\psi(z) .
$$


Substituting $z \rightarrow S T z$ in (4.4) and applying (4.3) repeatedly to get

$$
\begin{aligned}
2 \psi(T z) & =-(T z)^{\frac{d}{2}-2}\left(\psi\left(T^{-1} S T z\right)-2 \psi(S T z)+\psi(T S T z)\right) \\
& =\psi\left(T^{2} z\right)+2(T z)^{\frac{d}{2}-2} \psi(S T z)+\psi(z) \\
& =\psi\left(T^{2} z\right)-2 z^{\frac{d}{2}-2} \psi(S z)+\psi(z) \\
& =\psi\left(T^{2} z\right)+\psi(T z)-\psi(z)+\psi\left(T^{-1} z\right) .
\end{aligned}
$$

So, altogether we have that $\psi\left(T^{2} z\right)-\psi(T z)-\psi(z)+\psi\left(T^{-1} z\right)=0$. Defining $G(z):=\psi(T z)-\psi\left(T^{-1} z\right)$ implies $G(z+1)=G(z)$. Furthermore using (4.3) we obtain

$$
\begin{aligned}
z^{\frac{d}{2}-2} G(S z) & =z^{\frac{d}{2}-2}\left(\psi(T S z)-\psi\left(T^{-1} S z\right)\right) \\
& =-\psi\left(T^{-1} z\right)+\psi(T z) \\
& =G(z) .
\end{aligned}
$$

Therefore, $G$ is weakly holomorphic modular of weight $2-\frac{d}{2}$ for the full modular group using the growth condition (2.1). We now define

$$
\omega(z):=\psi(z)-\frac{1}{\pi i} G(z) \cdot \log \lambda(z)
$$

and from A.17) we see that $\omega$ is a weakly holomorphic modular form of weight $2-\frac{d}{2}$ for $\Gamma(2)$ (again using (2.1)). Moreover, plugging this relationship into (4.3) gives

$$
\omega(z)=z^{\frac{d}{2}-2} \omega(S z)+\omega(T z) .
$$

Finally, setting $f:=\frac{1}{\pi i} \cdot G$ we get the desired conclusions.

4.1. Determining $\psi$. In this step our goal will be determining $\psi$ given its representation in terms of $f$ and $\omega$. We use the fact that $\mathbb{C}(\lambda)$ is a field extension of $\mathbb{C}(j)$ to characterise the solutions of (4.10). Then using linear algebra, we ensure that conditions (2.1) and (2.7) hold. We will show that due to (4.10), achieving the former condition will give the latter.

To begin, we recall $f$ and $\omega$ are weakly holomorphic modular forms of weight $2-\frac{d}{2}$ for the groups $\Gamma$ and $\Gamma(2)$ respectively. There are no modular forms of negative weight because such forms must have poles on either $\mathbb{H}$ or at the cusps. The contour integration arguments from Proposition 2.2 rule out the former and so $f$ and $\omega$ must and can only have poles at the cusps. To continue, define

$$
\begin{aligned}
& \ell=\left\lceil\frac{d-4}{24}\right\rceil \\
& k=6 \ell-\frac{d-4}{4},
\end{aligned}
$$


which gives $0 \leq k \leq 5$. From this we set

$$
\begin{aligned}
& f=\frac{\omega_{k}}{\Delta^{\ell}} P^{(k)}(j) \\
& \omega=\frac{\omega_{k}}{\Delta^{\ell}} R^{(k)}(\lambda),
\end{aligned}
$$

where we recall from Table 3.1 that $\omega_{k}$ is a weakly holomorphic modular form for the full modular group of weight $2 k . P^{(k)}$ is a polynomial associated with each $k$, and $R^{(k)}$ is a rational function depending on our choice of $k$. This representation follows because $f \cdot \frac{\Delta^{\ell}}{\omega_{k}}$ is a weakly holomorphic form of weight 0 and using the fact that $j$ is Hauptmodul for $\Gamma$, this implies that it must be rational function in $j$. Moreover, since such a rational function can only have poles at $\infty$, it must be a polynomial. Analogously, since $\lambda$ is Hauptmodul for $\Gamma(2)$ we can similarly conclude that $\omega \cdot \frac{\Delta^{\ell}}{\omega_{k}}$ must be a rational function in $\lambda$. What differs here however is that $\Gamma(2)$ has three cusps (namely 0,1 , and $i \infty$ ). From our contour integration argument in Proposition 2.2 we see that we cannot have a pole at the origin (in fact (2.7) implies we must have a zero here), we can (in fact must) have a pole at $i \infty$, and we may have unprescribed behaviour at \pm 1 . This implies that the most we can conclude is that the denominator of such a rational function, say $R(x)$, can only have factors of the form $x^{a}(1-x)^{b}$ because $\lambda(0)=1$, $\lambda(1)=\infty$, and $\lambda(i \infty)=0$.

To continue, we will use (4.10) to analyse the possible choices for $R^{(k)}$. Combining (4.10) and (4.12) yields

$$
R^{(k)}(\lambda(z))=R^{(k)}(\lambda(S z))+R^{(k)}(\lambda(T z))
$$

We note that the field of meromorphic functions $\mathbb{C}(\lambda)$ is a degree 6 field extension over the field of meromorphic functions $\mathbb{C}(j)$ with the minimal polynomial of $\lambda$ over $\mathbb{C}(j)$ given by:

$$
\lambda^{6}-3 \lambda^{5}+(6-j) \lambda^{4}-(7-2 j) \lambda^{3}+(6-j) \lambda^{2}-3 \lambda+1=0
$$

Therefore, $R^{(k)}$ can be expressed in a unique way as

$$
R^{(k)}(\lambda)=\sum_{m=0}^{5} R_{m}^{(k)}(j) \lambda^{m}
$$

for rational functions $R_{m}^{(k)}$. Inserting this into (4.13) we get

$$
\sum_{m=0}^{5}\left((1-\lambda)^{5} \lambda^{m}-(1-\lambda)^{5+m}+(-1)^{m} \lambda^{m}(1-\lambda)^{5-m}\right) R_{m}^{(k)}(j)=0
$$

We can use the minimal polynomial (4.14) to write all powers of $\lambda$ larger than 5 by linear combinations of $\left\{1, \lambda, \ldots, \lambda^{5}\right\}$. This gives a linear system of 6 equations for the 6 unknown functions $R_{m}^{(k)}, k=0, \ldots, 5$. 
It can be checked directly that this system has rank 4 and hence has a 2 dimensional kernel. This supports an ansatz of the form

$$
\omega_{k} R^{(k)}(\lambda)=\chi_{1}^{(k)} Y^{(k)}(j)+\chi_{2}^{(k)} Z^{(k)}(j)
$$

where the $Y^{(k)}$ and $Z^{(k)}$ are polynomials and $\chi_{1}^{(k)}$ and $\chi_{2}^{(k)}$ are two linearly independent solutions of

$$
\chi(z)=z^{-2 k} \chi(S z)+\chi(T z) .
$$

Table 4.1 gives solutions of minimal orders at $z=0$ and $z=i \infty$.

\begin{tabular}{|c|c|c|}
\hline$k$ & $\chi_{1}^{(k)}$ & $\chi_{2}^{(k)}$ \\
\hline 0 & $\frac{(1+\lambda)(1-\lambda)\left(1-\lambda+\lambda^{2}\right)}{\lambda^{2}}$ & $\frac{(1+\lambda)\left(1-\lambda+\lambda^{2}\right)}{\lambda(1-\lambda)}$ \\
\hline 1 & $\theta_{00}^{4}(1-\lambda)$ & $\theta_{00}^{4} \frac{(1-\lambda)^{3}\left(2+3 \lambda+2 \lambda^{2}\right)}{\lambda^{2}}$ \\
\hline 2 & $\theta_{00}^{8}\left(1-\lambda^{2}\right)$ & $\theta_{00}^{8} \frac{(1+\lambda)\left(1+3 \lambda-7 \lambda^{2}+3 \lambda^{3}+\lambda^{4}\right)}{\lambda(1-\lambda)}$ \\
\hline 3 & $\theta_{00}^{12}(1-\lambda)\left(1-\lambda+\lambda^{2}\right)$ & $\theta_{00}^{12} \frac{\left(1-\lambda+\lambda^{2}\right)\left(1+3 \lambda-10 \lambda^{2}+3 \lambda^{3}+\lambda^{4}\right)}{\lambda(1-\lambda)}$ \\
\hline 4 & $\theta_{00}^{16} \lambda(1+\lambda)(1-\lambda)$ & $\theta_{00}^{16} \frac{(1+\lambda)\left(1-\lambda+\lambda^{2}-\lambda^{3}+\lambda^{4}-\lambda^{5}+\lambda^{6}\right)}{\lambda(1-\lambda)}$ \\
\hline 5 & $\theta_{00}^{20} \lambda(1-\lambda)\left(1-4 \lambda+\lambda^{2}\right)$ & $\theta_{00}^{20} \frac{1-32 \lambda^{3}+60 \lambda^{4}-32 \lambda^{5}+\lambda^{8}}{\lambda(1-\lambda)}$ \\
\hline
\end{tabular}

TABLE 4.1. The choices for the forms $\chi_{1}^{(k)}$ and $\chi_{2}^{(k)}$

Putting all this information together we get that $\psi$ has the form

$$
\psi=\frac{1}{\Delta^{\ell}}\left(X^{(k)}(j) \omega_{k} \log \lambda+\chi_{1}^{(k)} Y^{(k)}(j)+\chi_{2}^{(k)} Z^{(k)}(j)\right)
$$

for polynomials $X^{(k)}, Y^{(k)}, Z^{(k)}$ that depend on the value of $k$. Our next step will be to choose the degrees of $X^{(k)}, Y^{(k)}$, and $Z^{(k)}$ and use the degrees of freedom given by the coefficients so that (4.19) satisfies (2.7). In particular this implies that we need to choose their degrees so that $\psi$ vanishes to sufficiently large order at $i \infty$. In particular, we want

$$
\begin{aligned}
\varphi(z) & :=z^{-2 k}\left(X^{(k)}(j) \omega_{k}(S z) \log \lambda(S z)\right. \\
& \left.+\chi_{1}^{(k)}(S z) Y^{(k)}(j(z))+\chi_{2}^{(k)}(S z) Z^{(k)}(j(z))\right)=\mathcal{O}\left(q^{\ell+\frac{1}{2}}\right) .
\end{aligned}
$$

Before continuing in this direction however, we show two short lemmas.

Lemma 4.2. Suppose $\varphi(z)$ is as in (4.20). Then it has only half integer exponents in its Fourier expansion.

Proof. Let

$$
\left.\chi(z)=\chi_{1}^{(k)}(z) Y^{(k)}(j(z))+\chi_{2}^{(k)}(z) Z^{(k)}(j(z))\right),
$$


denote sum of the last two terms on the right side of (4.20). Then $\chi$ satisfies (4.18) and so

$$
z^{-2 k} \chi(S z)=\chi(T z)-\chi(z)
$$

which implies that all terms in the Fourier expansion of $z^{-2 k} \chi(S z)$ with integer exponents vanish. Moreover, we see from (A.19) that the expression $z^{-2 k} X^{(k)}(j) \omega_{k}(S z) \log \lambda(S z)$ has only half integer exponents in its Fourier expansion, giving the claim.

Lemma 4.3. Let $\psi$ be given by (4.19) with polynomials $X, Y, Z$ such that (2.7) holds. Then the principal part of $\psi$ at $i \infty$ has only integer exponents of $q$.

Proof. By our assumption $z^{\frac{d}{2}-2} \psi(S z)=\mathcal{O}\left(q^{\frac{1}{2}}\right)$. Since $\psi$ can be written as

$$
\psi(z)=\sum_{k=-m}^{\infty} a_{k} q^{\frac{k}{2}}-i z \sum_{k=-n}^{\infty} b_{k} q^{k}=\psi_{1}+z \psi_{2},
$$

(4.4) implies that $\psi_{1}$ satisfies

$$
\begin{aligned}
\psi(T z)-2 \psi(z)+\psi\left(T^{-1} z\right)=\psi_{1}(T z) & -2 \psi_{1}(z)+\psi_{1}\left(T^{-1} z\right) \\
& =2 \psi_{1}(T z)-2 \psi_{1}(z)=\mathcal{O}\left(q^{\frac{1}{2}}\right),
\end{aligned}
$$

which gives the assertion of the lemma.

In light of Lemmas 4.2 and 4.3, we first assume that (2.7) holds and define the subscript $n$ for the polynomial $X_{n}^{(k)}$ so that the following polar order is achieved.

$$
X_{n}^{(k)}(j) \omega_{k}=\mathcal{O}\left(q^{-n}\right),
$$

We note that this implies that for each $k \neq 1$ the degree of the polynomial $X_{n}^{(k)}$ is at most $n$ and for $k=1$ that it has degree at most $n-1$. We similarly adopt the notations $Y_{n}^{(k)}$ and $Z_{n}^{(k)}$ to refer to the polynomials that give us:

$$
\begin{aligned}
\chi_{1}^{(k)}(z) Y_{n}^{(k)}(j(z))+\chi_{2}^{(k)}(z) Z_{n}^{(k)}(j(z)) & =\mathcal{O}\left(q^{-n-1}\right) \\
z^{-2 k}\left(\chi_{1}^{(k)}(S z) Y_{n}^{(k)}(j(z))\right) & =\mathcal{O}\left(q^{-n+\frac{1}{2}}\right) \\
z^{-2 k}\left(\chi_{2}^{(k)}(S z) Z_{n}^{(k)}(j(z))\right) & =\mathcal{O}\left(q^{-n+\frac{1}{2}}\right) .
\end{aligned}
$$

We observe that (4.22), (4.23), and (4.24) are sufficient to put upper bounds on the degrees of polynomials $Y_{n}^{(k)}$ and $Z_{n}^{(k)}$. With $b(k)$ as in Table 4.2 we can use the degrees of freedom gained from the coefficients of $X_{n}^{(k)}, Y_{n}^{(k)}$, and $Z_{n}^{(k)}$ so that

$$
\begin{aligned}
& z^{-2 k}\left(X_{n}^{(k)}(j) \omega_{k}(S z) \log \lambda(S z)+\chi_{1}^{(k)}(S z) Y_{n}^{(k)}(j(z))\right. \\
& \left.+\chi_{2}^{(k)}(S z) Z_{n}^{(k)}(j(z))\right)=\mathcal{O}\left(q^{2 n+\frac{b(k)}{2}}\right)
\end{aligned}
$$


which is a strengthening of our hypothesis that (2.7) is satisfied. We then observe that (4.21) and (4.22) ensure by (2.3) that the largest real second order pole of $W(s)$ is 2 less than the largest real first order pole. Altogether, this will give us the desired sign change of the function $F$ given by (2.6). The degrees of these polynomials are also detailed in Table 4.2 .

\begin{tabular}{|c|c|c|c|c|}
\hline$k$ & $\operatorname{deg} X_{n}^{(k)}$ & $\operatorname{deg} Y_{n}^{(k)}$ & $\operatorname{deg} Z_{n}^{(k)}$ & $b(k)$ \\
\hline 0 & $n$ & $n$ & $n-1$ & 3 \\
\hline 1 & $n-1$ & $n$ & $n$ & 3 \\
\hline 2 & $n$ & $n+1$ & $n$ & 5 \\
\hline 3 & $n$ & $n+1$ & $n$ & 5 \\
\hline 4 & $n$ & $n+2$ & $n+1$ & 7 \\
\hline 5 & $n$ & $n+2$ & $n+1$ & 7 \\
\hline
\end{tabular}

TABLE 4.2. Degrees of the polynomials $X_{n}^{(k)}, Y_{n}^{(k)}$, and $Z_{n}^{(k)}$

We now need to choose $n$ so that

$$
2 n+\frac{b(k)}{2}>\ell
$$

so that (2.7) is satisfied. This then gives that the minimal choice of $n$ is then

$$
n=\left\lceil\frac{2 \ell-b(k)}{4}\right\rceil .
$$

Then conditions (4.21) and (4.22) ensure that there is a sign change of $F(\mathbf{x})$ at $\|\mathbf{x}\|^{2}=2 n+2 \ell+2$ and $F(\mathbf{x}) \neq 0$ for $\|\mathbf{x}\|^{2}=2 n+2 \ell$. Expressing $\ell, k$, and $n$ in terms of $d$ yields $2 n+2 \ell=2\left\lfloor\frac{d}{16}\right\rfloor$.

Summing up, we have proved most of the following theorem. The theorem is formulated with some abuse of notation, which is justified by the fact that it discusses radial functions: we write $F_{-}(\mathbf{x})=F_{-}(\|\mathbf{x}\|)$ and consider $F_{-}$as multivariate and univariate function as appropriate.

Theorem 4.4. For $d \equiv 0(\bmod 4)$ set $n_{-}=\left\lfloor\frac{d}{16}\right\rfloor+1$. Then there exists a radial Schwartz function $F_{-}: \mathbb{R}^{d} \rightarrow \mathbb{R}$ satisfying

$$
\begin{aligned}
F_{-}(\mathbf{x}) & =(-1)^{\frac{d}{4}+1} \widehat{F}_{-}(\mathbf{x}) \quad \text { for all } \mathbf{x} \in \mathbb{R}^{d} \\
F_{-}\left(\sqrt{2 n_{-}}\right) & =0 \quad \text { and } F_{-}^{\prime}\left(\sqrt{2 n_{-}}\right) \neq 0 \\
F_{-}(\sqrt{2 m}) & =F_{-}^{\prime}(\sqrt{2 m})=0 \quad \text { for } m>n_{-}, \quad m \in \mathbb{N} .
\end{aligned}
$$

Proof. It remains to show that $F_{-}^{\prime}\left(\sqrt{2 n_{-}}\right) \neq 0$. This follows from the discussion of vanishing orders in Section 5.2, especially equation (5.24) and the discussion following. More precisely, (5.24) implies that $a_{n_{-}} \neq 0$ in (2.1), whereas (5.25) implies that $b_{n_{-}}=0$. This shows by 


\begin{tabular}{|l|r|r|r|l|l|l|}
\cline { 2 - 7 } \multicolumn{1}{c|}{} & \multicolumn{2}{|c|}{$d$} & $n$ & $X_{n}^{(k)}(w)$ & $Y_{n}^{(k)}(w)$ & $Z_{n}^{(k)}(w)$ \\
\hline \multirow{2}{*}{$k=0$} & 4 & 28 & 0 & 2 & 1 & 0 \\
\cline { 2 - 7 } & 52 & 76 & 1 & $120 \times(7 w+384)$ & $63 w+171776$ & 91392 \\
\hline \hline \multirow{2}{*}{$k=1$} & & 24 & 0 & 0 & 0 & 1 \\
\cline { 2 - 7 } & 48 & 72 & 1 & 840 & $514304-840 w$ & $63 w+131584$ \\
\hline \hline \multirow{3}{*}{$k=2$} & 20 & 44 & 0 & 6144 & $5 w+8192$ & -1280 \\
\cline { 2 - 7 } & 68 & 92 & 1 & $53760 \times(3 w+$ & $33 w^{2}+$ & $-202688 w+$ \\
& & & & $512)$ & 117014528 & $88256)$ \\
\hline \hline \multirow{3}{*}{$k=3$} & 16 & 40 & 0 & 1536 & $5 w-9856$ & 640 \\
\cline { 2 - 7 } & 64 & 88 & 1 & $215040 \times(3 w+$ & $231 w^{2}-$ & $128 \times(231 w+$ \\
& & & & $128)$ & $26752 w-$ & $1002752)$ \\
\hline \hline \multirow{3}{*}{$k=4$} & & 12 & -1 & 0 & $w+768$ & -256 \\
\cline { 2 - 7 } & 36 & 60 & 0 & 7864320 & $-7 w^{2}-14080 w-$ & $256(7 w+8704)$ \\
\hline \hline \multirow{2}{*}{$k=5$} & & 8 & -1 & 0 & 3670016 & \\
\cline { 2 - 7 } & 32 & 56 & 0 & 55050240 & $-35 w^{2}-$ & $256 \times(35 w-$ \\
& & & & & 89587712 & $29824)$ \\
\hline
\end{tabular}

TABLE 4.3. The polynomials $X_{n}^{(k)}, Y_{n}^{(k)}$, and $Z_{n}^{(k)}$; the dimensions in the left column are those covered by Remark (4.29).

(2.3) that $W$ has a simple pole at $s=2 n_{-}$, which gives a simple zero of $U$ at this point.

Remark 4.5. Notice that by (4.25) the order increases by 2 , if $n$ increases by 1 . Together with (4.27) this gives that for certain $d(\bmod 48)$ the order of vanishing of $\psi$ is one more than required. This means that for these values of the dimension there is one extra degree of freedom. This is the case for dimensions

$$
d \equiv\{0,4,16,20,32,36\} \quad(\bmod 48) .
$$

\section{Modular Differential Equations}

In Section 3.1 we discussed the existence of the form $\psi(S z) z^{\frac{d}{2}-2}$ given by (3.1) in Proposition 3.1 by a simple dimension count. A similar argument was used in Section 4.1 to obtain certain modular forms for $\Gamma(2)$ that satisfy the requirements of Proposition 4.1. In the present section we give a direct construction by an explicit linear recurrence, which we obtain via modular differential equations. For this purpose we set

$$
f_{12 n+2 k+4}=\Delta^{n}\left(\omega_{k+2} P_{n}^{(k)}(j)-2 \omega_{k+1} E_{2} Q_{n}^{(k)}(j)+\omega_{k} E_{2}^{2} R_{n}^{(k)}(j)\right),
$$


where $P_{n}^{(k)}, Q_{n}^{(k)}$, and $R_{n}^{(k)}$ are the polynomials used in the ansatz (3.12) studied in Section 3.1. Similarly, we set

$$
\phi_{12 n+2 k+12}=\Delta^{n+1}\left(\omega_{k} X_{n}^{(k)} \log \lambda+\chi_{1}^{(k)} Y_{n}^{(k)}(j)+\chi_{2}^{(k)} Z_{n}^{(k)}(j)\right),
$$

where $X_{n}^{(k)}, Y_{n}^{(k)}$, and $Z_{n}^{(k)}$ are the polynomials used in the ansatz (4.19) in Section 4.1. In this section we use the weight $w=12 n+2 k+4$ of the form $f_{w}$ and $w=12 n+2 k+12$ of the form $\phi_{w}$ as the parameter.

5.1. Differential equations for quasimodular forms. The quasimodular form $f_{w}$ of weight $w$ and depth 2 is then given by the three requirements

$$
\begin{aligned}
& f_{w}=\mathcal{O}\left(q^{3 n+a(k)-1}\right)=\mathcal{O}\left(q^{\left\lfloor\frac{w}{4}\right\rfloor-1}\right) \\
& g_{w}=\Delta^{n}\left(\omega_{k+1} Q_{n}^{(k)}(j)-\omega_{k} E_{2} R_{n}^{(k)}(j)\right)=\mathcal{O}(q) \\
& h_{w}=\Delta^{n} \omega_{k} R_{n}^{(k)}(j)=\mathcal{O}(1) .
\end{aligned}
$$

The third equation (5.5) is of course trivially satisfied, we mention it only, because we will show in the sequel that (5.3) -(5.5) give the exact order for $z \rightarrow i \infty$ of the functions $f_{w}, g_{w}$, and $h_{w}$ obtained by our construction and that the solution is uniquely characterised by these conditions. We notice that forms satisfying $f_{w}=\mathcal{O}\left(q^{\left\lfloor^{\left.\frac{w}{4}\right\rfloor}\right.}\right)$ are called extremal quasimodular forms; they were studied in [23, 34. We will adapt the methods used in these two papers to our situation.

In [24] third order linear differential equations are characterised, whose solution set is invariant under the modular group. Especially, several cases of third order linear differential equations are shown to have modular or quasimodular solutions. In [18] modular differential equations of orders $\leq 5$ having quasimodular solutions are fully characterised. Such differential equations are especially useful for finding quasimodular forms $f_{w}$ with prescribed behaviour of $f_{w}, g_{w}$ and $h_{w}$ for $q \rightarrow 0$, because the corresponding orders have to be solutions of the indicial equation. In this section we will present this approach to finding the quasimodular forms satisfying the requirements of Proposition 3.1.

Assuming the defining properties of the forms $f_{w}$ for even weights $w \geq 8$, there exist coefficients $a_{w}, b_{w}, c_{w}$ such that

$$
f_{w+4}=a_{w} E_{4} f_{w}+b_{w} E_{4}^{2} f_{w-4}+c_{w} \Delta f_{w-8} .
$$

This comes from considering the orders of the three forms on the right hand side: the last two terms have the same orders for $q \rightarrow 0$, thus the coefficients $b_{w}$ and $c_{w}$ can be chosen so that the order of the sum $b_{w} E_{4}^{2} f_{w-4}+c_{w} \Delta f_{w-8}$ equals the order of $E_{4} f_{w}$. Then $a_{w}$ can be chosen to again increase the order by 1 . Since the corresponding functions $g_{w}$ and $h_{w}$ satisfy the same recurrence relation, these functions automatically satisfy (5.4) and (5.5) by induction. At this moment this argument involves some heuristics, namely that there is no higher order of vanishing in any term than expected. We will make this rigorous 
by showing that the solutions of the linear recurrence satisfy certain differential equations, which show that they have precisely the assumed order of vanishing. This will then allow us to find values for $a_{w}, b_{w}$, and $c_{w}$ and suitable initial values for $f_{w}$.

For the purpose of the proof we recall the definition of the Serre derivative (see [36])

$$
\partial_{w} f=f^{\prime}-\frac{w}{12} E_{2} f .
$$

Notice that the Serre derivative $\partial_{w}$ applied to a modular form of weight $w$ gives a modular form of weight $w+2$; similarly, the Serre derivative $\partial_{w-2}$ applied to a quasimodular form of weight $w$ and depth 2 gives a quasimodular form of weight $w+2$ and depth $\leq 2$; this can be seen from

$$
\begin{aligned}
& \partial_{w-2}\left(A_{w}+E_{2} B_{w-2}+E_{2}^{2} C_{w-4}\right)=\partial_{w} A_{w}-\frac{1}{12} E_{4} B_{w-2} \\
+ & E_{2}\left(\frac{1}{6} A_{w}+\partial_{w-2} B_{w-2}-\frac{1}{6} E_{4} C_{w-4}\right) \\
+ & E_{2}^{2}\left(\frac{1}{12} B_{w-2}+\partial_{w-4} C_{w-4}\right)
\end{aligned}
$$

where $A_{w}, B_{w-2}$, and $C_{w-4}$ are modular forms of respective weights $w$, $w-2$, and $w-4$.

Furthermore, we recall the definition of the Rankin-Cohen bracket (see [35])

$$
[f, g]_{n}^{(k, \ell)}=\sum_{i=0}^{n}(-1)^{i}\left(\begin{array}{c}
n+k-1 \\
n-i
\end{array}\right)\left(\begin{array}{c}
n+\ell-1 \\
i
\end{array}\right) f^{(i)} g^{(n-i)}
$$

The cases $w \equiv 0(\bmod 4)$ (this corresponds to $k=0,2,4)$ and $w \equiv 2$ $(\bmod 4)$ (this corresponds to $k=1,3,5)$ have to be treated slightly differently. In the second case the underlying modular differential equation takes a somehow non-standard form, which was not covered by the general study [24].

Proposition 5.1. Consider the sequence $\left(f_{w}\right)_{w},(w \equiv 0(\bmod 4), w \geq$ 8) given by the initial elements

$$
\begin{aligned}
f_{8} & =E_{4}^{2}-2 E_{2} E_{6}+E_{2}^{2} E_{4}=\frac{36}{5} E_{4}^{\prime \prime} \\
f_{12} & =\frac{1}{6000}\left(E_{6}^{2}-2 E_{2} E_{4} E_{6}+E_{2}^{2} E_{4}^{2}\right)=\frac{1}{3000}\left(E_{4}^{2}\right)^{\prime \prime}-\frac{4}{25} \Delta \\
f_{16} & =\frac{1}{2540160000}\left(-25 E_{4}^{4}+49 E_{4} E_{6}^{2}-48 E_{2} E_{4}^{2} E_{6}+E_{2}^{2}\left(49 E_{4}^{3}-25 E_{6}^{2}\right)\right) \\
& =\frac{\left(25 E_{6}^{2}-49 E_{4}^{3}\right)^{\prime \prime}}{2751840000}-\frac{\Delta E_{4}}{45500}
\end{aligned}
$$


and the recurrence

$$
\begin{aligned}
f_{w+4} & =\frac{1}{16000(w+2)(w-3)(w-5)(w-9)(w-10)(w-11)} \\
& \times\left(200(w-8)(w-9)\left(w^{2}-15 w+38\right) E_{4} f_{w}\right. \\
& \left.-\frac{5}{8}(w-8)(w-12) E_{4}^{2} f_{w-4}+\Delta f_{w-8}\right) .
\end{aligned}
$$

Then for every $w \equiv 0(\bmod 4), w \geq 8$ the quasimodular form $f_{w}$ satisfies (5.3) and the corresponding functions $g_{w}$ and $h_{w}$ satisfy (5.4) and (5.5), where $q^{\frac{w}{4}-1}, q$, and 1 are the exact orders of these functions for $q \rightarrow 0$.

Proof. We will prove the proposition by showing that $f_{w}$ satisfies the differential equation

$$
\begin{aligned}
f^{\prime \prime \prime} & -\frac{w}{4} E_{2} f^{\prime \prime}+\left(\frac{w-4}{4} E_{4}+\frac{w(w-1)}{4} E_{2}^{\prime}\right) f^{\prime} \\
& -\left(\frac{(w-2)(w-4)}{16} E_{4}^{\prime}+\frac{w(w-1)(w-2)}{24} E_{2}^{\prime \prime}\right) f=0 .
\end{aligned}
$$

This is the case $\alpha=\frac{w-4}{4}$ and $\beta=0$ of the general form of linear differential equations admitting modular and quasimodular solutions given in [24, Theorem 1]. We will write (5.12) $)_{w}$ to indicate the dependence on the parameter $w\left(\right.$ so, $f_{w-4}$ is a solution of $\left.(5.12) w-4\right)$. The indicial equation of (5.12) then reads as

$$
\mu^{3}-\frac{w}{4} \mu^{2}+\frac{w-4}{4} \mu=\mu(\mu-1)\left(\mu-\frac{w-4}{4}\right)=0 .
$$

The roots of this equation correspond to the exponents in (5.3)-(5.5) .

The differential equation (5.12) can be rewritten in terms of the Serre derivative as

$$
\begin{aligned}
\partial_{w+2} \partial_{w} \partial_{w-2} f & -\frac{3 w^{2}-36 w+140}{144} E_{4} \partial_{w-2} f \\
& -\frac{(w-2)(w-5)(w-14)}{864} E_{6} f=0 .
\end{aligned}
$$

The following lemma can be checked by direct computation.

Lemma 5.2. If $F_{w}$ is a solution of $(\underline{5.12})$, then the function

$$
\frac{1}{\Delta}\left(\left[F_{w}, E_{4}\right]_{2}^{(w-2,4)}+\frac{5}{3}\left[F_{w}, E_{6}\right]_{1}^{(w-2,6)}\right)
$$

is a solution of $(\underline{5.12})_{w-4}$.

We now proceed by induction to show that the right-hand-side of (5.11) is a solution of $(5.12) w+4$. Assume that we have proved that $f_{m}$ 
is a solution of $(5.12) m$ and that

$$
\Delta f_{m-4}=\left[f_{m}, E_{4}\right]_{2}^{(m-2,4)}+\frac{5}{3}\left[f_{m}, E_{6}\right]_{1}^{(m-2,6)}
$$

for $12 \leq m \leq w, m \equiv 0(\bmod 4)$. Inserting $(\underline{5.16})$ for $m=w-4$ and $m=w$ into (5.11) and using that $f_{w-4}$ and $f_{w}$ are solutions of $(5.12) w_{w-4}$ and $(5.12) w$ gives that the right-hand-side of (5.11) equals

$$
\frac{(w-5)(w-6) E_{4} f_{w}-36 \partial_{w} \partial_{w-2} f_{w}}{120(w+2)(w-3)(w-5)(w-10)} .
$$

This can be checked to be a solution of $(5.12) w+4$. In order to complete the induction step, we verify (5.16) for $m=w+4$ inserting the expression (5.17). This shows that indeed (5.11) holds.

The solution of the recurrence (5.11) is thus also a solution of the differential equation (5.12). Since $f_{w}$ vanishes to at least second order in $q$ for $q \rightarrow 0$, it can only be the solution of (5.12) associated to the root $\mu=\frac{w-4}{4}$ of the indicial equation. Thus (5.3) holds. Similarly, since $g_{w}$ and $h_{w}$ satisfy the same linear recurrence relation, we prove that (5.4) and (5.5) hold.

Proposition 5.3. Consider the sequence $\left(f_{w}\right)_{w},(w \equiv 2(\bmod 4), w \geq$ 10) given by the initial elements

$$
\begin{aligned}
f_{10} & =-E_{4} E_{6}+2 E_{2} E_{4}^{2}-E_{2}^{2} E_{6}=-\frac{24}{7} E_{6}^{\prime \prime} \\
f_{14} & =-\frac{1}{8400}\left(E_{4}^{2} E_{6}-E_{2}\left(E_{4}^{3}+E_{6}^{2}\right)+E_{2}^{2} E_{4} E_{6}\right) \\
& =-\frac{3}{19250}\left(E_{4} E_{6}\right)^{\prime \prime}-\frac{36}{875} E_{2} \Delta \\
f_{18} & =-\frac{1}{237600}\left(5 E_{4}^{3} E_{6}+7 E_{6}^{3}-E_{2}\left(5 E_{4}^{4}+19 E_{4} E_{6}^{2}\right)+12 E_{2}^{2} E_{4}^{2} E_{6}\right) \\
& =-\frac{1}{28875}\left(E_{4}^{2} E_{6}\right)^{\prime \prime}+\frac{2 \Delta\left(181 E_{6}-185 E_{2} E_{4}\right)}{9625}
\end{aligned}
$$

and the recurrence

$$
\begin{aligned}
f_{w+4} & =\frac{1}{16000(w-3)(w-4)(w-5)(w-9)(w-11)(w-16)} \\
& \times\left(200(w-9)(w-10)\left(w^{2}-21 w+92\right) E_{4} f_{w}\right. \\
& \left.-\frac{5}{8}(w-10)(w-14) E_{4}^{2} f_{w-4}+\Delta f_{w-8}\right) .
\end{aligned}
$$

Then for every $w \equiv 2(\bmod 4), w \geq 10$ the quasimodular form $f_{w}$ satisfies (5.3) and the corresponding functions $g_{w}$ and $h_{w}$ satisfy (5.4) and (5.5), where $q^{\frac{w-6}{4}}, q$, and 1 are the exact orders of these functions for $q \rightarrow 0$. 
Proof. We will prove the proposition by showing that $f_{w}$ satisfies the differential equation

$$
\begin{aligned}
& E_{6} f^{\prime \prime \prime}-\left(\frac{w-2}{4} E_{4}^{2}+\frac{w}{2} E_{6}^{\prime}\right) f^{\prime \prime} \\
+ & \left(\frac{w-6}{4} E_{4} E_{6}+\frac{(w-1)(w-2)}{8} E_{4} E_{4}^{\prime}+\frac{w(w-1)}{14} E_{6}^{\prime \prime}\right) f^{\prime} \\
- & \left(\frac{(w-2)(w-6)}{24} E_{4} E_{6}^{\prime}+\frac{5(w-8)(w-9)(w-10)}{384}\left(E_{4}^{\prime}\right)^{2}\right. \\
& \left.+\frac{w^{3}+105 w^{2}-1162 w+3576}{480} E_{4} E_{4}^{\prime \prime}+\frac{w(w-1)(w-2)}{336} E_{6}^{\prime \prime \prime}\right) f=0 .
\end{aligned}
$$

The extra factor $E_{6}$ in front of the highest derivative is motivated by the computations in [34]. The indicial equation is then given by

$$
\mu^{3}-\frac{w-2}{4} \mu^{2}+\frac{w-6}{4} \mu=\mu(\mu-1)\left(\mu-\frac{w-6}{4}\right)=0 .
$$

The equation (5.20) can be written in terms of Serre derivatives as

$$
\begin{aligned}
& E_{6} \partial_{w+2} \partial_{w} \partial_{w-2} f+\frac{1}{2} E_{4}^{2} \partial_{w} \partial_{w-2} f-\frac{3 w^{2}-48 w+224}{144} E_{4} E_{6} \partial_{w-2} f \\
& -\left(\frac{3 w^{2}-48 w+224}{288} E_{4}^{3}+\frac{w^{3}-33 w^{2}+300 w-896}{864} E_{6}^{2}\right) f=0
\end{aligned}
$$

Then a relation similar to Lemma 5.2 holds.

Lemma 5.4. If $F_{w}$ is a solution of $(5.20){ }_{w}$, then the function

$$
\frac{1}{\Delta}\left(\left[F_{w}, E_{4}\right]_{2}^{(w-2,4)}+\frac{5}{3}\left[F_{w}, E_{6}\right]_{1}^{(w-2,6)}\right)
$$

is a solution of $(\underline{5.20})_{w-4}$.

We proceed in a similar manner as in the proof of Proposition 5.1. The main equation after inserting (5.22) into the recurrence and using that $f_{w}$ is a solution of (5.20) reads

$$
f_{w+4}=\frac{(w-8)(w-9) E_{4} f_{w}-36 \partial_{w} \partial_{w-2} f_{w}}{120(w-3)(w-4)(w-5)(w-16)}
$$

This can be checked again to satisfy $(5.20) \mathrm{w}+4$ and $(5.16) w+4$. The remaining arguments are also similar. 
5.2. Differential equations for $\Gamma(2)$-modular forms. The forms $\phi_{w}$ are given by the requirements

$$
\begin{aligned}
\phi_{w}(z) & =\mathcal{O}(1) \\
\phi_{w}(z)-\phi_{w}(T z) & =\mathcal{O}(q) \\
z^{-w} \phi_{w}(S z) & =\mathcal{O}\left(q^{\left\lfloor\frac{w}{4}\right\rfloor-\frac{1}{2}}\right) .
\end{aligned}
$$

Notice that (5.25) is equivalent to $f \Delta^{n+1}$, with $f$ as in (4.1), being a cusp form. Furthermore, we will show that the orders of vanishing for $z \rightarrow i \infty$ given in (5.24) - (5.26) are exact. Similarly to (5.6) we expect a linear recurrence equation for $\phi_{w}$ by the same heuristic argument. The fact that such recurrences indeed hold, is the content of the following two propositions. Their proofs follow exactly the same lines as the proofs of Propositions 5.1 and 5.3 .

Proposition 5.5. Consider the sequence $\left(\phi_{w}\right)_{w},(w \equiv 0(\bmod 4), w \geq$ 8) given by the initial elements

$$
\begin{aligned}
\phi_{8} & =\theta_{01}^{12}\left(\theta_{01}^{4}+2 \theta_{10}^{4}\right) \\
\phi_{12} & =\frac{8}{175} \Delta \log \lambda+\frac{\theta_{01}^{12}}{11200}\left(2 \theta_{10}^{12}+3 \theta_{01}^{4} \theta_{10}^{8}+3 \theta_{01}^{8} \theta_{10}^{4}+\theta_{01}^{12}\right) \\
\phi_{16} & =\frac{1}{231000} \Delta E_{4} \log \lambda \\
& +\frac{\theta_{01}^{12}}{1419264000}\left(24 \theta_{10}^{20}+60 \theta_{01}^{4} \theta_{10}^{16}+68 \theta_{01}^{8} \theta_{10}^{12}+42 \theta_{01}^{12} \theta_{10}^{8}\right)
\end{aligned}
$$

and the recurrence (for $w \geq 16$ )

$$
\begin{aligned}
\phi_{w+4} & =\frac{1}{16000(w+4)(w-1)(w-3)(w-7)(w-8)(w-9)} \\
& \times\left(200(w-6)(w-7)\left(w^{2}-11 w+12\right) E_{4} \phi_{w}\right. \\
& \left.-\frac{5}{8}(w-6)(w-10) E_{4}^{2} \phi_{w-4}+\Delta \phi_{w-8}\right) .
\end{aligned}
$$

Then for every $w \equiv 0(\bmod 4), w \geq 8$ the modular form $\phi_{w}$ satisfies (5.24), (5.25), and (5.26), where $1, q$, and $q^{\frac{w-2}{4}}$ are the exact orders of these functions for $q \rightarrow 0$.

Proof. As pointed out, the proof follows the same lines as the proof of Proposition 5.1; it only has to be shown that $\phi_{w}$ satisfies $(5.12) w+2$. This is done by showing the relation

$$
\phi_{w+4}=\frac{(w-3)(w-4) E_{4} \phi_{w}-36 \partial_{w+2} \partial_{w} \phi_{w}}{120(w+4)(w-1)(w-3)(w-8)} .
$$


Proposition 5.6. Consider the sequence $\left(\phi_{w}\right)_{w},(w \equiv 2(\bmod 4), w \geq$ 10) given by the initial elements

$$
\begin{aligned}
\phi_{10} & =\theta_{01}^{12}\left(5 \theta_{10}^{8}+5 \theta_{01}^{4} \theta_{10}^{4}+2 \theta_{01}^{8}\right) \\
\phi_{14} & =\frac{\theta_{01}^{20}}{13440}\left(7 \theta_{10}^{8}+7 \theta_{01}^{4} \theta_{10}^{4}+2 \theta_{01}^{8}\right) \\
\phi_{18}= & \frac{1}{600600} \Delta E_{6} \log \lambda \\
+ & \frac{\theta_{01}^{12}}{1845043200}\left(-12 \theta_{10}^{24}-36 \theta_{01}^{4} \theta_{10}^{20}-13 \theta_{01}^{8} \theta_{10}^{16}+34 \theta_{01}^{12} \theta_{10}^{12}\right. \\
& \left.\quad+68 \theta_{01}^{16} \theta_{10}^{8}+45 \theta_{01}^{20} \theta_{10}^{4}+10 \theta_{01}^{24}\right)
\end{aligned}
$$

and the recurrence (for $w \geq 18$ )

$$
\begin{aligned}
\phi_{w+4} & =\frac{1}{16000(w-1)(w-2)(w-3)(w-7)(w-9)(w-14)} \\
& \times\left(200(w-7)(w-8)\left(w^{2}-17 w+54\right) E_{4} \phi_{w}\right. \\
& \left.-\frac{5}{8}(w-8)(w-12) E_{4}^{2} \phi_{w-4}+\Delta \phi_{w-8}\right) .
\end{aligned}
$$

Then for every $w \equiv 2(\bmod 4), w \geq 10$ the modular form $\phi_{w}$ satisfies (5.24), (5.25), and (5.26), where $1, q$, and $q^{\frac{w-4}{4}}$ are the exact orders of these functions for $q \rightarrow 0$.

Proof. The proof is again similar to the proof of Proposition 5.3, it only has to be shown that $\phi_{w}$ satisfies (5.20) $w+2$. This is done by showing the relation

$$
\phi_{w+4}=\frac{(w-6)(w-9) E_{4} \phi_{w}-36 \partial_{w+2} \partial_{w} \phi_{w}}{120(w-1)(w-2)(w-3)(w-14)} .
$$

\section{Positivity of the Coefficients}

In this section we will show that the forms $f_{w}$ obtained in Propositions 5.1 and 5.3 can be written in the form

$$
f_{w}=(-1)^{w / 2} \frac{144 \mu_{w}}{(w-3)(w-4)} E_{w-4}^{\prime \prime}+\Delta \tilde{f}_{w-12}
$$

where $\tilde{f}_{w-12}$ is a quasimodular form of weight $w-12$ and depth 2 . The factor $\mu_{w}$ is given by

$$
\mu_{w}=\left\{\begin{array}{lll}
\frac{3(w / 4-2) !}{80^{w / 4-2}(w-7) ! !(w / 2-1) ! !} & \text { for } w \equiv 0 & (\bmod 4) \\
\frac{3((w-10) / 4) !}{80^{(w-10) / 4}(w-7) ! !(w / 2-4) ! !} & \text { for } w \equiv 2 & (\bmod 4)
\end{array}\right.
$$

where $m ! !=m(m-2)(m-4) \ldots$ denotes the double factorial. From this we derive the following proposition. 
Proposition 6.1. Let $f_{w}$ be the quasimodular forms given by Propositions 5.1 and 5.3. Then all Fourier coefficients of $f_{w}$ are positive with possibly finitely many exceptions.

Proof. We set

$$
f_{w}=A_{w}+E_{2} B_{w-2}+E_{2}^{2} C_{w-4} .
$$

Applying (5.8) twice we obtain

$$
\begin{aligned}
& \partial_{w} \partial_{w-2}\left(A_{w}+E_{2} B_{w-2}+E_{2}^{2} C_{w-4}\right)= \\
& \frac{1}{72}\left(72 \partial_{w+2} \partial_{w} A_{w}-E_{4} A_{w}-12 E_{4} \partial_{w-2} B_{w-2}+2 E_{6} B_{w-2}+E_{4}^{2} C_{w-4}\right) \\
+ & \frac{E_{2}}{36}\left(12 \partial_{w} A_{w}+\partial_{w} \partial_{w-2} B_{w-2}-E_{4} B_{w-2}-12 E_{4} \partial_{w-4} C_{w-4}+2 E_{6} C_{w-4}\right) \\
+ & \frac{E_{2}^{2}}{72}\left(A_{w}+12 \partial_{w-2} B_{w-2}+72 \partial_{w-2} \partial_{w-4} C_{w-4}-E_{4} C_{w-4}\right) .
\end{aligned}
$$

Setting $a_{w}, b_{w-2}$, and $c_{w-4}$ for the constant coefficients of $A_{w}, B_{w-2}$, and $C_{w-4}$ and using (5.17) we obtain

$$
\left(\begin{array}{l}
a_{w+4} \\
b_{w+2} \\
c_{w}
\end{array}\right)=\frac{\left(\begin{array}{ccc}
3 w^{2}-46 w+122 & -2 w & -2 \\
4 w & 3 w^{2}-42 w+124 & 8-4 w \\
-2 & 2(w-2) & 3 w^{2}-38 w+114
\end{array}\right)\left(\begin{array}{l}
a_{w} \\
b_{w-2} \\
c_{w-4}
\end{array}\right)}{480(w-10)(w-5)(w-3)(w+2)}
$$

from which we obtain

$$
\left(\begin{array}{l}
a_{w} \\
b_{w-2} \\
c_{w-4}
\end{array}\right)=\mu_{w}\left(\begin{array}{r}
1 \\
-2 \\
1
\end{array}\right)
$$

for $w \equiv 0(\bmod 4)$; using $(5.23)$ for $w \equiv 2(\bmod 4)$ gives a similar recursion. This shows that all three forms $A_{w}, B_{w-2}$, and $C_{w-4}$ have non-vanishing Eisenstein parts.

The form $f_{w}$ can be rewritten as

$$
f_{w}=\alpha_{w-4}^{\prime \prime}+\beta_{w-2}^{\prime}+\gamma_{w}
$$

with forms $\alpha_{w-4}, \beta_{w-2}$, and $\gamma_{w}$ of respective weights $w-4, w-2$, and $w$. The two representations (6.3) and (6.5) are related by

$$
\begin{aligned}
\alpha_{w-4} & =\frac{144}{(w-3)(w-4)} C_{w-4} \\
\beta_{w-2} & =\frac{12}{w-2}\left(B_{w-2}-\frac{24}{w-4} \partial_{w-4} C_{w-4}\right) \\
\gamma_{w} & =A_{w}-\frac{12}{w-2} \partial_{w-2} B_{w-2}+\frac{1}{w-3} E_{4} C_{w-4} \\
& +\frac{144}{(w-2)(w-3)} \partial_{w-2} \partial_{w-4} C_{w-4} .
\end{aligned}
$$


Inserting our previous result that the constant coefficients of $A_{w}, B_{w-2}$, and $C_{w-4}$ are proportional to $(1,-2,1)$ yields that $\beta_{w-2}$ and $\gamma_{w}$ are cusp forms, thus multiples of $\Delta$. This yields the decomposition (6.1) and more precisely

$$
f_{w}=(-1)^{w / 2} \frac{144 \mu_{w}}{(w-3)(w-4)} E_{w-4}^{\prime \prime}+\widetilde{\alpha}_{w-4}^{\prime \prime}+\beta_{w-2}^{\prime}+\gamma_{w}
$$

with cusp forms $\widetilde{\alpha}_{w-4}, \beta_{w-2}$, and $\gamma_{w}$.

The Fourier coefficients of $E_{w-4}^{\prime \prime}$ are $-\frac{2(w-4)}{\mathcal{B}_{w-4}} n^{2} \sigma_{w-5}(n)$ and thus of order $n^{w-3}$ (here $\mathcal{B}_{w-4}$ denotes the Bernoulli numbers). Using Deligne's estimate (see [14]) for the coefficients of cusp forms to estimate the coefficients of $\widetilde{\alpha}_{w-4}^{\prime \prime}+\beta_{w-2}^{\prime}+\gamma_{w}$ gives an estimate of order $n^{\frac{w-1}{2}} \sigma_{0}(n)$ $\left(\sigma_{0}(n)\right.$ being the number of positive divisors of $\left.n\right)$ for the coefficients of $\Delta \tilde{f}_{w-12}$ in (6.1). Thus the coefficient of the second term in (6.1) is of smaller order than the coefficient of the first term, which then determines the sign of all but possibly finitely many Fourier coefficients.

We explain shortly, how to prove positivity of all coefficients for a fixed value of $w$ : we start from (6.7). Then the coefficients of the cusp forms can be estimated by an explicit bound obtained in [21].

Theorem 6.2 (Theorem 1 in [21]). Let

$$
G(z)=\sum_{n=1}^{\infty} g(n) q^{n}
$$

be a cusp form of weight $w$. Then

$$
\begin{aligned}
|g(n)| & \leq \sqrt{\log w}\left(11 \sqrt{\sum_{m=1}^{\ell} \frac{|g(m)|^{2}}{m^{w-1}}}\right. \\
& \left.+\frac{e^{18.72}(41.41)^{w / 2}}{w^{(w-1) / 2}}\left|\sum_{m=1}^{\ell} g(m) e^{-7.288 m}\right|\right) n^{\frac{w-1}{2}} \sigma_{0}(n),
\end{aligned}
$$

where $\ell$ is the dimension of the space of cusp forms of weight $w$.

Using this bound we obtain that the coefficients of $\widetilde{\alpha}_{w-4}^{\prime \prime}, \beta_{w-2}^{\prime}$, and $\gamma_{w}$ are bounded by

$$
C_{i} n^{\frac{w-1}{2}} \sigma_{0}(n) \text { for } i=\alpha, \beta, \gamma
$$

for explicit constants $C_{\alpha}, C_{\beta}$, and $C_{\gamma}$. Then the coefficients of $f_{w}$ are positive for all $n$ satisfying

$$
\frac{144 \mu_{w}}{(w-3)(w-4)} \frac{2(w-4)}{\left|\mathcal{B}_{w-4}\right|} n^{2} \sigma_{w-5}(n)>\left(C_{\alpha}+C_{\beta}+C_{\gamma}\right) n^{\frac{w-1}{2}} \sigma_{0}(n) .
$$

Estimating $\sigma_{w-5}(n) \geq n^{w-5}$ and $\sigma_{0}(n)<2 \sqrt{n}$, this can be solved explicitly for the minimal value of $n$; the remaining finitely many values 
of $n$ can be checked with the help of a computer. The number of coefficients to be checked was up to 3300 in the cases we studied. For the values $w \leq 22$ the cusp forms are either trivial or Hecke eigenforms, because the space of cusp forms has dimension $\leq 1$. In this case Deligne's estimate can be used directly, and the number of coefficients to be checked is less than 10 .

Remark 6.3. We have checked the positivity of the Fourier coefficients of the forms $f_{w}$ for even $w$ in the range $8, \ldots, 94$, which corresponds to dimensions $d=4, \ldots, 312$ ( $d$ divisible by 4 ). Notice that the weight depends on dimension by the relation

$$
w=12\left\lfloor\frac{d+4}{16}\right\rfloor-\frac{d}{2}+16 .
$$

As pointed out in Theorem 3.2 this implies that $F_{+}(\mathbf{x}) \leq 0$ for $\|\mathbf{x}\|^{2} \geq$ $2 n+2 \ell-2$ for these dimensions.

The numerical experiments support the following conjecture.

Conjecture 1. Let $f_{w}(w \equiv 0(\bmod 2))$ be the quasimodular form given by Propositions 5.1 and 5.3. Then

$$
f_{w}=\sum_{m=\left\lfloor\frac{w}{4}\right\rfloor-1}^{\infty} a_{w}(m) q^{m}
$$

with $a_{w}(m)>0$ for $m \geq\left\lfloor\frac{w}{4}\right\rfloor-1$.

This conjecture is similar to Conjecture 2 in [23], which states the positivity of the Fourier coefficients of extremal quasimodular forms of depth $\leq 4$.

Remark 6.4. The Fourier coefficients of the modular forms $\phi_{w}$ do not seem to be positive for small values of $w$. Numerical experiments indicate that the functions $\phi_{w}(i t)$ are positive for $t>0$. Except for $w=8,10,14$ (corresponding to dimensions 8, 12, and 24) this seems to be difficult to prove due to the presence of the $\log \lambda$-term.

\section{EXAMPLES: SMALL DIMENSIONS}

For some small dimensions the functions we constructed in Sections 3.1 and 4.1 are of special interest. These of course include the dimensions 8, 12, and 24 studied in [10, 11, 32]. In the following we express all $\Gamma(2)$-modular functions in terms of $\theta$-functions by replacing $\lambda$ using (A.11).

Dimension 4 . The function $\lambda$ maps the positive imaginary axis to the interval $(0,1)$. Thus the function $\log \lambda$ produces a non-positive +1 eigenfunction for the Fourier transform. The function $\chi_{1}^{(0)}$ turns into $\mathrm{a}+1$ eigenfunction vanishing at $\mathbf{0}$ and having a last sign change at distance $\sqrt{2}$. This shows $A_{+}(4) \leq \sqrt{2}$ 
The function

$$
8400 \frac{f_{14}}{\Delta}=\frac{E_{4}^{2} E_{6}-E_{2}\left(E_{4}^{3}+E_{6}^{2}\right)+E_{2}^{2} E_{4} E_{6}}{\Delta}
$$

produces a Fourier eigenfunction for the eigenvalue -1 , which does not vanish at $\mathbf{0}$ and has a last sign change at distance $\sqrt{2}$. This shows $A_{-}(4) \leq \sqrt{2}$.

Dimension 8. Our results recover the functions used in [32] to prove the optimal upper bound for sphere packings in dimension 8: the quasimodular form used there is

$$
6000 \frac{f_{12}}{\Delta}=\frac{E_{6}^{2}-2 E_{2} E_{4} E_{6}+E_{2}^{2} E_{4}^{2}}{\Delta} .
$$

The second modular form giving the -1 Fourier eigenfunction is

$$
\frac{\phi_{10}}{\Delta}=\frac{\theta_{01}^{12}\left(5 \theta_{10}^{8}+5 \theta_{01}^{4} \theta_{10}^{4}+2 \theta_{01}^{8}\right)}{\Delta} .
$$

Dimension 12. Similarly, the function used in [10] to show an optimal uncertainty principle in dimension 12 is given by

$$
\frac{\phi_{8}}{\Delta}=\frac{\theta_{01}^{12}\left(\theta_{01}^{4}+2 \theta_{10}^{4}\right)}{\Delta}
$$

The function

$$
\begin{aligned}
C_{22} \frac{f_{22}}{\Delta^{2}}=\frac{1}{\Delta^{2}}\left(205 E_{4}^{4} E_{6}-637 E_{4} E_{6}^{3}+E_{2}\right. & \left.70 E_{4}^{5}+794 E_{4}^{2} E_{6}^{2}\right) \\
+ & \left.E_{2}^{2}\left(275 E_{6}^{3}-707 E_{4}^{3} E_{6}\right)\right)
\end{aligned}
$$

( $C_{22}$ being some large integer constant) provides a -1 eigenfunction, which does not vanish at $\mathbf{0}$ and has a last sign change at 2 . In this case Remark 3.3 applies and we can achieve a more suitable function by forming a linear combination of $f_{22}$ and $E_{4} f_{18}$ to obtain the function

$$
\begin{aligned}
\frac{1}{\Delta^{2}}\left(415 E_{4}^{4} E_{6}+161 E_{4} E_{6}^{3}\right)-2 E_{2}\left(431 E_{4}^{2} E_{6}^{2}+145 E_{4}^{5}\right) \\
\left.+E_{2}^{2}\left(451 E_{4}^{3} E_{6}+125 E_{6}^{3}\right)\right),
\end{aligned}
$$

which transforms into an eigenfunction vanishing at $\mathbf{0}$ and having a last sign change at distance 2 . This shows $A_{-}(12) \leq 2$.

Dimension 16. This is the smallest dimension for which both Remarks 3.3 and 4.5 apply.

The function

$$
\begin{aligned}
C_{20} \frac{f_{20}}{\Delta^{2}}=\frac{1}{\Delta^{2}}\left(-469 E_{4}^{2} E_{6}^{2}+325 E_{4}^{5}+E_{2}\right. & \left(358 E_{4}^{3} E_{6}-70 E_{6}^{3}\right) \\
& \left.+E_{2}^{2}\left(395 E_{4} E_{6}^{2}-539 E_{4}^{4}\right)\right)
\end{aligned}
$$


transforms into a Fourier eigenfunction for the eigenvalue 1, which has a last sign change at distance 2. By Remark 3.3 we have an extra degree of freedom by forming a linear combination of $f_{20}$ and $E_{4} f_{16}$ to obtain the function

$$
\begin{aligned}
\frac{1}{\Delta^{2}}\left(203 E_{4}^{2} E_{6}^{2}+85 E_{4}^{5}-2 E_{2}\left(211 E_{4}^{3} E_{6}\right.\right. & \left.+77 E_{6}^{3}\right) \\
& \left.+E_{2}^{2}\left(239 E_{4} E_{6}^{2}+49 E_{4}^{4}\right)\right),
\end{aligned}
$$

which transforms into a Fourier eigenfunction vanishing at $\mathbf{0}$ and having a last sign change at distance 2 . This shows $A_{+}(16) \leq 2$.

The functions

$$
\begin{aligned}
C_{18} \frac{\phi_{18}}{\Delta^{2}}=\frac{3072 E_{6}}{\Delta} \log \lambda+ & \frac{\theta_{01}^{12}}{\Delta^{2}}\left(-12 \theta_{10}^{24}-36 \theta_{01}^{4} \theta_{10}^{20}-13 \theta_{01}^{8} \theta_{10}^{16}\right. \\
& \left.+34 \theta_{01}^{12} \theta_{10}^{12}+68 \theta_{01}^{16} \theta_{10}^{8}+45 \theta_{01}^{20} \theta_{10}^{4}+10 \theta_{01}^{24}\right)
\end{aligned}
$$

and

$$
C_{14} \frac{E_{4} \phi_{14}}{\Delta^{2}}=\frac{\theta_{01}^{4}\left(\theta_{01}^{8}+\theta_{01}^{4} \theta_{10}^{4}+\theta_{10}^{8}\right)\left(7 \theta_{10}^{8}+7 \theta_{01}^{4} \theta_{10}^{4}+2 \theta_{01}^{8}\right)}{\theta_{10}^{16} \theta_{00}^{16}}
$$

both transform into Fourier eigenfunctions with last sign change at distance 2. The transform of the second function vanishes at $\mathbf{0}$. This shows $A_{-}(16) \leq 2$.

Dimension 20. The function

$$
C_{18} \frac{f_{18}}{\Delta^{2}}=\frac{1}{\Delta^{2}}\left(5 E_{4}^{3} E_{6}+7 E_{6}^{3}+E_{2}\left(-19 E_{4} E_{6}^{2}-5 E_{4}^{4}\right)+12 E_{2}^{2} E_{4}^{2} E_{6}\right)
$$

transforms into a Fourier eigenfunction for eigenvalue -1 , which has its last sign change at distance 2 . This eigenfunction does not vanish at $\mathbf{0}$. This shows $A_{-}(20) \leq 2$.

The functions

$$
\begin{aligned}
C_{16} & \frac{\phi_{16}}{\Delta^{2}}=3 \frac{E_{4}}{\Delta} \log \lambda \\
& +\frac{32\left(5 \theta_{01}^{20}+20 \theta_{01}^{16} \theta_{10}^{4}+42 \theta_{01}^{12} \theta_{10}^{8}+68 \theta_{01}^{8} \theta_{10}^{12}+60 \theta_{01}^{4} \theta_{10}^{16}+24 \theta_{10}^{20}\right)}{\theta_{01}^{4} \theta_{10}^{16} \theta_{00}^{16}}
\end{aligned}
$$

and

$$
C_{12} \frac{E_{4} \phi_{12}}{\Delta^{2}}=\frac{E_{4}}{\Delta} \log \lambda+\frac{128\left(\theta_{01}^{4}+2 \theta_{10}^{4}\right)\left(\theta_{01}^{8}+\theta_{01}^{4} \theta_{10}^{4}+\theta_{10}^{8}\right)^{2}}{\theta_{01}^{4} \theta_{10}^{16} \theta_{00}^{16}}
$$

both transform into Fourier eigenfunctions with a last sign change at distance 2. Forming the linear combination

$$
\frac{\theta_{01}^{4}\left(\theta_{01}^{12}+4 \theta_{01}^{8} \theta_{10}^{4}+6 \theta_{01}^{4} \theta_{10}^{8}+4 \theta_{10}^{12}\right)}{\theta_{10}^{16} \theta_{00}^{16}}
$$


gives a function, which transforms into a Fourier eigenfunction vanishing at $\mathbf{0}$ and having sign changes exactly at distances $\sqrt{2}$ and 2 . This shows $A_{+}(20) \leq 2$.

Dimension 24. The functions used in [11] to obtain the optimal upper bound for sphere packings in dimension 24 were

$$
2540160000 \frac{f_{16}}{\Delta^{2}}=\frac{-25 E_{4}^{4}+49 E_{4} E_{6}^{2}-48 E_{2} E_{4}^{2} E_{6}+E_{2}^{2}\left(49 E_{4}^{3}-25 E_{6}^{2}\right)}{\Delta^{2}}
$$

and

$$
13440 \frac{\phi_{14}}{\Delta^{2}}=\frac{\theta_{01}^{20}\left(7 \theta_{10}^{8}+7 \theta_{01}^{4} \theta_{10}^{4}+2 \theta_{01}^{8}\right)}{\Delta^{2}} .
$$

Dimension 48. The function

$$
\begin{aligned}
C_{28} \frac{f_{28}}{\Delta^{4}} & =\frac{1}{\Delta^{4}}\left(-9565298 E_{4}^{4} E_{6}^{2}+1101373 E_{4} E_{6}^{4}+7254325 E_{4}^{7}\right. \\
& +48 E_{2}\left(133387 E_{4}^{5} E_{6}-82987 E_{4}^{2} E_{6}^{3}\right) \\
& \left.+E_{2}^{2}\left(10650578 E_{4}^{3} E_{6}^{2}-11603053 E_{4}^{6}-257125 E_{6}^{4}\right)\right)
\end{aligned}
$$

( $C_{28}$ being some large integer constant) transforms into a Fourier eigenfunction for the eigenvalue 1 , which has a last sign change at distance $\sqrt{8}$. In this case Remark 3.3 applies and we can achieve a more suitable function by forming a linear combination of $f_{28}$ and $E_{4} f_{24}$ to obtain the function

$$
\begin{aligned}
\frac{1}{\Delta^{4}}(-216724 & 8 E_{4}^{4} E_{6}^{2}+29822429 E_{4} E_{6}^{4}+16373825 E_{4}^{7} \\
& +24 E_{2}\left(53329 E_{4}^{5} E_{6}-2096977 E_{4}^{2} E_{6}^{3}\right) \\
+ & \left.E_{2}^{2}\left(58616494 E_{4}^{3} E_{6}^{2}-23223893 E_{4}^{6}-10868825 E_{6}^{4}\right)\right)
\end{aligned}
$$

which transforms into a Fourier eigenfunction vanishing at $\mathbf{0}$ with last sign change at $\sqrt{8}$. This shows $A_{+}(48) \leq \sqrt{8}$

The functions

$$
\begin{aligned}
C_{26} \frac{\phi_{26}}{\Delta^{3}} & =267 \frac{E_{4}^{2} E_{6}}{\Delta^{2}} \log \lambda+\frac{2048}{\theta_{01}^{12} \theta_{10}^{24} \theta_{00}^{24}} \\
& \times\left(570 \theta_{01}^{40}+3705 \theta_{01}^{36} \theta_{10}^{4}+14204 \theta_{01}^{32} \theta_{10}^{8}+41639 \theta_{01}^{28} \theta_{10}^{12}\right. \\
+ & 71710 \theta_{01}^{24} \theta_{10}^{16}+50531 \theta_{01}^{20} \theta_{10}^{20}-26351 \theta_{01}^{16} \theta_{10}^{24}-88288 \theta_{01}^{12} \theta_{10}^{28} \\
& \left.-86152 \theta_{01}^{8} \theta_{10}^{32}-42720 \theta_{01}^{4} \theta_{10}^{36}-8544 \theta_{10}^{40}\right)
\end{aligned}
$$


and

$$
\begin{aligned}
& C_{22} \frac{E_{4} \phi_{22}}{\Delta^{3}}=3 \frac{E_{4}^{2} E_{6}}{\Delta^{2}} \log \lambda+\frac{1024\left(\theta_{01}^{8}+\theta_{01}^{4} \theta_{10}^{4}+\theta_{10}^{8}\right)}{\theta_{01}^{12} \theta_{10}^{24} \theta_{00}^{24}} \\
& \times\left(30 \theta_{01}^{32}+165 \theta_{01}^{28} \theta_{10}^{4}+395 \theta_{01}^{24} \theta_{10}^{8}+636 \theta_{01}^{20} \theta_{10}^{12}+566 \theta_{01}^{16} \theta_{10}^{16}-240 \theta_{01}^{12} \theta_{10}^{20}\right. \\
& \left.-976 \theta_{01}^{8} \theta_{10}^{24}-768 \theta_{01}^{4} \theta_{10}^{28}-192 \theta_{10}^{32}\right)
\end{aligned}
$$

transform into Fourier eigenfunctions with a last sign change at distance $\sqrt{6}$. The linear combination

$$
\begin{aligned}
\frac{\theta_{01}^{16}}{\theta_{10}^{24} \theta_{00}^{24}}\left(170 \theta_{01}^{24}+\right. & 1105 \theta_{01}^{20} \theta_{10}^{4}+2678 \theta_{01}^{16} \theta_{10}^{8} \\
+ & \left.2574 \theta_{01}^{12} \theta_{10}^{12}-143 \theta_{01}^{8} \theta_{10}^{16}-1716 \theta_{01}^{4} \theta_{10}^{20}-572 \theta_{10}^{24}\right)
\end{aligned}
$$

of these two functions transforms into a Fourier eigenfunction vanishing at $\mathbf{0}$ with sign changes exactly at distances $\sqrt{2}, 2$, and $\sqrt{6}$. This shows $A_{-}(48) \leq \sqrt{6}$.

This should be compared to [28]. There it is erroneously stated that for dimension 48 only a +1 eigenfunction with last sign change at distance $\sqrt{6}$ can be obtained by this method. Our result shows that this method produces a -1 eigenfunction with last sign change at $\sqrt{6}$, whereas the optimal +1 eigenfunction obtained by this method has its last sign change at distance $\sqrt{8}$.

\section{Appendix A. Some PREliminaries on modular FORMS AND FUNCTIONS}

In this appendix we provide some basic facts about modular and quasimodular forms, which are required as background for Sections 3 and 4. For further reading and more details we refer to [4] and [6, 15, 20, 30,31]. For introductions to quasimodular forms we refer to [5, 29, 36].

We recall the definition of the modular group

$$
\Gamma=\operatorname{PSL}(2, \mathbb{Z})=\operatorname{SL}(2, \mathbb{Z}) /\{ \pm I\}
$$

and the congruence subgroup

$$
\Gamma(2)=\{\gamma \in \operatorname{SL}(2, \mathbb{Z}) \mid \gamma \equiv I \quad(\bmod 2)\} /\{ \pm I\},
$$

where $I$ denotes the identity matrix. We also recall that $\Gamma$ is generated by $T: z \mapsto z+1$ and $S: z \mapsto-\frac{1}{z} ; \Gamma(2)$ is generated by $S T^{2} S=\frac{-z}{2 z-1}$ and $T^{2}: z \mapsto z+2$.

A.1. Modular forms for $\Gamma$, Eisenstein series. A holomorphic function $f: \mathbb{H} \rightarrow \mathbb{C}$ is called a weakly holomorphic modular form of weight $k$, if it satisfies

$$
(c z+d)^{-k} f\left(\frac{a z+b}{c z+d}\right)=f(z)
$$


for all $z \in \mathbb{H}$ and all $\left(\begin{array}{ll}a & b \\ c & d\end{array}\right) \in \Gamma$. The space of weakly holomorphic modular forms is denoted by $\mathcal{M}_{k}^{!}(\Gamma)$. This space is non-trivial only for even values of $k$. A form $f$ is called holomorphic, if

$$
f(i \infty):=\lim _{\Im z \rightarrow+\infty} f(z)
$$

exists. The subspace $\mathcal{M}_{k}(\Gamma)$ of holomorphic modular forms is nontrivial only for even $k \geq 4$. Its dimension equals

$$
\operatorname{dim} \mathcal{M}_{k}(\Gamma)= \begin{cases}\left\lfloor\frac{k}{12}\right\rfloor & \text { for } k \equiv 2 \quad(\bmod 12) \\ \left\lfloor\frac{k}{12}\right\rfloor+1 & \text { otherwise. }\end{cases}
$$

A holomorphic form $f$ is called a cusp form, if $f(i \infty)=0$. The space of cusp forms is denoted by $\mathcal{S}_{k}(\Gamma)$.

Prototypical examples of modular forms of weight $2 k$ are given by the Eisenstein series

$$
E_{2 k}(z)=\frac{1}{2 \zeta(2 k)} \sum_{(m, n) \in \mathbb{Z} \backslash\{(0,0)\}} \frac{1}{(m z+n)^{2 k}}=1-\frac{4 k}{\mathcal{B}_{2 k}} \sum_{n=1}^{\infty} \sigma_{2 k-1}(n) q^{n}
$$

for $k \geq 2$ with

$$
\sigma_{2 k-1}(n)=\sum_{d \mid n} d^{2 k-1}
$$

and $\mathcal{B}_{2 k}$ denoting the Bernoulli numbers. As usual in the context of modular forms we use the notation $q=e^{2 \pi i z} ; q$ is called the nome.

The two forms

$$
E_{4}(z)=1+240 \sum_{n=1}^{\infty} \sigma_{3}(n) q^{n}, \quad E_{6}(z)=1-504 \sum_{n=1}^{\infty} \sigma_{5}(n) q^{n},
$$

of respective weights 4 and 6 are especially important, since they generate the ring of all holomorphic modular forms

$$
\bigoplus_{k=0}^{\infty} \mathcal{M}_{2 k}(\Gamma)=\mathbb{C}\left[E_{4}, E_{6}\right]
$$

The modular discriminant is the prototype of a cusp form

$$
\Delta(z)=\frac{1}{1728}\left(E_{4}(z)^{3}-E_{6}(z)^{2}\right)=q \prod_{n=1}^{\infty}\left(1-q^{n}\right)^{24} .
$$

Its weight is 12 . The relation

$$
\mathcal{S}_{2 k}(\Gamma)=\Delta \mathcal{M}_{2 k-12}(\Gamma)
$$

characterises the spaces of cusp forms. Furthermore, we have

$$
\mathcal{M}_{2 k}(\Gamma)=\mathbb{C} E_{2 k} \oplus \mathcal{S}_{2 k}(\Gamma) .
$$

This decomposition is used in Section 6 to split forms into an Eisenstein part (a form with non-vanishing constant coefficient, for instance a multiple of $\left.E_{2 k}\right)$ and a cusp form. 
Klein's modular function

$$
j(z)=\frac{E_{4}(z)^{3}}{\Delta(z)}
$$

generates the field of all modular functions (forms of weight 0)

$\{f: \mathbb{H} \rightarrow \mathbb{C} \mid \forall \gamma \in \Gamma: f(\gamma z)=f(z)$ and $f$ meromorphic $\}=\mathbb{C}(j)$.

This fact is expressed by calling $j$ a Hauptmodul for $\Gamma$.

A.2. Modular forms for $\Gamma(2)$, Theta series. Powers of the Jacobi theta functions

$$
\begin{aligned}
& \theta_{00}(z)=\sum_{n=-\infty}^{\infty} e^{\pi i n^{2} z} \\
& \theta_{01}(z)=\sum_{n=-\infty}^{\infty}(-1)^{n} e^{\pi i n^{2} z} \\
& \theta_{10}(z)=\sum_{n=-\infty}^{\infty} e^{\pi i\left(n+\frac{1}{2}\right)^{2} z}
\end{aligned}
$$

are examples of modular forms for $\Gamma(2)$. From these definitions it follows that

$$
\begin{aligned}
& \theta_{00}(z)^{4}=1+\sum_{n=1}^{\infty} r_{4}(n) q^{\frac{n}{2}} \\
& \theta_{01}(z)^{4}=1+\sum_{n=1}^{\infty}(-1)^{n} r_{4}(n) q^{\frac{n}{2}}
\end{aligned}
$$

where

$$
r_{4}(k):=\left|\left\{\mathbf{x} \in \mathbb{Z}^{4} \mid\|\mathbf{x}\|^{2}=k\right\}\right|,
$$

denotes the number of possibilities to express $n$ as a sum of four squares. Jacobi's famous four-square theorem gives the following representation of $r_{4}(k)$

$$
r_{4}(k)=8 \sigma_{1}(k)-32 \sigma_{1}\left(\frac{k}{4}\right),
$$

where we use the convention for arithmetic functions that they are defined to be 0 for non-integer arguments.

The $\theta$-functions satisfy the transformation formulas

$$
\begin{array}{ll}
\theta_{00}(T z)^{4}=\theta_{01}(z)^{4}, & z^{-2} \theta_{00}(S z)^{4}=-\theta_{00}(z) \\
\theta_{01}(T z)^{4}=\theta_{00}(z)^{4}, & z^{-2} \theta_{01}(S z)^{4}=-\theta_{10}(z)^{4} \\
\theta_{10}(T z)^{4}=-\theta_{10}(z)^{4}, & z^{-2} \theta_{10}(S z)^{4}=-\theta_{01}(z)^{4}
\end{array}
$$

and Jacobi's famous relation

$$
\theta_{01}(z)^{4}+\theta_{10}(z)^{4}=\theta_{00}(z)^{4}
$$


The modular $\lambda$-function

$$
\lambda(z)=\frac{\theta_{10}(z)^{4}}{\theta_{00}(z)^{4}}=\frac{\theta_{10}(z)^{4}}{\theta_{01}(z)^{4}+\theta_{10}(z)^{4}}
$$

is a Hauptmodul for $\Gamma(2)$ and satisfies

$$
j=256 \frac{\left(1-\lambda+\lambda^{2}\right)^{3}}{\lambda^{2}(1-\lambda)^{2}} .
$$

The fact that

$$
[\mathbb{C}(\lambda): \mathbb{C}(j)]=6
$$

is used in Section 4. The following transformation formulas follow from (A.9)

$$
\begin{aligned}
& \lambda(T z)=\frac{\lambda(z)}{\lambda(z)-1} \\
& \lambda(S z)=1-\lambda(z) .
\end{aligned}
$$

The function $\lambda$ is holomorphic on $\mathbb{H}$, attains the value 1 at $z=0$, and has no zeros in $\mathbb{H}$. Hence, we may define a holomorphic logarithm of $\lambda$ by

$$
\log \lambda(z):=2 \pi i \int_{0}^{z} \frac{\lambda^{\prime}(w)}{\lambda(w)} d w=\pi i \int_{0}^{z} \theta_{01}^{4}(w) d w
$$

where the second equation follows from (A.23). Notice that $\log \lambda(i t) \in$ $\mathbb{R}_{<0}$ for $t \in \mathbb{R}_{>0}$. We observe via direct computation with the contour integral and the properties of $\lambda$ that:

$$
\begin{aligned}
\log \lambda\left(T^{2} z\right) & =\log \lambda(z)+2 \pi i \\
2 \log \lambda(S z) & =\log \lambda\left(T^{-1} z\right)-2 \log \lambda(z)+\log \lambda(T z) .
\end{aligned}
$$

Notice that these equations imply

$$
\log \lambda(z)=\log \lambda(T z)+\log \lambda(S z)+\pi i
$$

which is used in Section 4.

Using the second equality of (A.14) and (A.6) we obtain the following expansion of $\log \lambda$ at the cusp $i \infty$ :

$$
\log \lambda(z)=\pi i z+4 \log (2)+\sum_{k=1}^{\infty}(-1)^{k} \frac{r_{4}(k)}{k} q^{\frac{k}{2}},
$$

where $r_{4}$ is defined in (A.7). Then (A.16), (A.18) together with (A.8) give the expansion

$$
\log \lambda(S z)=-16 \sum_{k=0}^{\infty} \frac{\sigma_{1}(2 k+1)}{2 k+1} q^{k+\frac{1}{2}} .
$$




\section{A.3. Derivatives of modular forms and quasimodular forms.}

The ring of modular forms is not closed under differentiation, which can be seen by differentiating (A.1). Notice that it is common and convenient in the context of modular forms to set $f^{\prime}=\frac{1}{2 \pi i} \frac{d f}{d z}=q \frac{d f}{d q}$. By adjoining the Eisenstein series of weight 2

$$
E_{2}(z)=1-24 \sum_{n=1}^{\infty} \sigma_{1}(n) q^{n}
$$

which satisfies the transformation formula

$$
z^{-2} E_{2}(S z)=E_{2}(z)+\frac{6}{\pi i z}
$$

we obtain the ring $\mathbb{C}\left[E_{2}, E_{4}, E_{6}\right]$ of quasimodular forms, which is closed under differentiation. This can be seen from Ramanujan's identities

$$
\begin{aligned}
E_{2}^{\prime} & =\frac{1}{12}\left(E_{2}^{2}-E_{4}\right) \\
E_{4}^{\prime} & =\frac{1}{3}\left(E_{2} E_{4}-E_{6}\right) \\
E_{6}^{\prime} & =\frac{1}{2}\left(E_{2} E_{6}-E_{4}^{2}\right) .
\end{aligned}
$$

Furthermore, we have

$$
j^{\prime}=-\frac{E_{4}^{2} E_{6}}{\Delta}=-\frac{E_{6}}{E_{4}} j
$$

and

$$
\lambda^{\prime}(z)=\frac{1}{2} \theta_{01}^{4}(z) \lambda(z)
$$

as well as

$$
\begin{aligned}
& \left(\theta_{00}^{4}\right)^{\prime}=\frac{1}{6}\left(E_{2} \theta_{00}^{4}-\theta_{01}^{8}+\theta_{10}^{8}\right) \\
& \left(\theta_{01}^{4}\right)^{\prime}=\frac{1}{6}\left(E_{2} \theta_{01}^{4}-\theta_{01}^{8}-2 \theta_{01}^{4} \theta_{10}^{4}\right) \\
& \left(\theta_{10}^{4}\right)^{\prime}=\frac{1}{6}\left(E_{2} \theta_{10}^{4}+2 \theta_{01}^{4} \theta_{10}^{4}+\theta_{10}^{8}\right) .
\end{aligned}
$$

A quasimodular form $f$ of weight $2 k$ can be written as

$$
f(z)=\sum_{\ell=0}^{k} E_{2}^{\ell}(z) f_{2 k-2 \ell}(z)
$$

where $f_{2 k-2 \ell}$ is a modular form of weight $2 k-2 \ell$; the term for $\ell=k-1$ is of course trivial. Quasimodular forms are invariant under $T$ and transform under $S$ by

$$
z^{-2 k} f(S z)=\sum_{m=0}^{k}\left(\frac{6}{\pi i z}\right)^{m} \sum_{\ell=0}^{k-m}\left(\begin{array}{c}
m+\ell \\
\ell
\end{array}\right) E_{2}^{\ell}(z) f_{2 k-2 \ell-2 m}(z) .
$$


Notice that the terms

$$
\sum_{\ell=0}^{k-m}\left(\begin{array}{c}
m+\ell \\
\ell
\end{array}\right) E_{2}^{\ell}(z) f_{2 k-2 \ell-2 m}(z)
$$

are quasimodular forms of weight $2 k-2 m$. The largest value $\ell$, for which $f_{2 k-2 \ell}$ in (A.25) is non-zero is called the depth of the quasimodular form.

The derivative of a quasimodular form of weight $2 k$ and depth $s$ is a quasimodular form of weight $2 k+2$ and depth at most $s+1$. In particular, the derivative of a modular form of weight $2 k$ is a quasimodular form of weight $2 k+2$ and depth 1 for $k>0$.

Acknowledgement. This material is based upon work supported by the National Science Foundation under Grant No. DMS-1439786 while the authors were in residence at the Institute for Computational and Experimental Research in Mathematics in Providence, RI, during the Spring 2018 semester.

The second author is grateful to Masanobu Kaneko for very helpful email conversations and for providing the Master thesis [34].

The authors are grateful to the anonymous referee for her/his valuable suggestions which increased the quality of this paper.

\section{REFERENCES}

1. T. Aste and D. Weaire, The pursuit of perfect packing, second ed., Taylor \& Francis, New York, 2008.

2. R. P. Boas, Jr., Entire functions, Academic Press Inc., New York, 1954.

3. J. Bourgain, L. Clozel, and J.-P. Kahane, Principe d'Heisenberg et fonctions positives, Ann. Inst. Fourier (Grenoble) 60 (2010), no. 4, 1215-1232.

4. J. H. Bruinier, G. van der Geer, G. Harder, and D. Zagier, The 1-2-3 of modular forms, Universitext, Springer-Verlag, Berlin, 2008, Lectures from the Summer School on Modular Forms and their Applications held in Nordfjordeid, June 2004, Edited by Kristian Ranestad.

5. Y. J. Choie and M. H. Lee, Jacobi-Like Forms, Pseudodifferential Operators, and Quasimodular Forms, Monographs in Mathematics, Springer International Publishing, 2019.

6. H. Cohen and F. Strömberg, Modular forms, Graduate Studies in Mathematics, vol. 179, American Mathematical Society, Providence, RI, 2017, A classical approach.

7. H. Cohn, New upper bounds on sphere packings. II, Geom. Topol. 6 (2002), 329-353.

8. 64 (2017), no. 2, 102-115.

9. H. Cohn and N. Elkies, New upper bounds on sphere packings. I, Ann. of Math. (2) 157 (2003), no. 2, 689-714.

10. H. Cohn and F. Gonçalves, An optimal uncertainty principle in twelve dimensions via modular forms, Invent. Math. 217 (2019), no. 3, 799-831.

11. H. Cohn, A. Kumar, S. D. Miller, D. Radchenko, and M. Viazovska, The sphere packing problem in dimension 24, Ann. of Math. (2) 185 (2017), no. 3, 10171033 . 
12. Universal optimality of the $E_{8}$ and Leech lattices and interpolation formulas, https://arxiv.org/abs/1902.05438, Feb 2019.

13. D. de Laat and F. Vallentin, A breakthrough in sphere packing: the search for magic functions, Nieuw Arch. Wiskd. (5) 17 (2016), no. 3, 184-192, Includes an interview with Henry Cohn, Abhinav Kumar, Stephen D. Miller and Maryna Viazovska.

14. P. Deligne, La conjecture de Weil, Publ. Math. I.H.E.S. 43 (1974), 273-307.

15. F. Diamond and J. Shurman, A first course in modular forms, Graduate Texts in Mathematics, vol. 228, Springer-Verlag, New York, 2005.

16. L. Fejes, Über die dichteste Kugellagerung, Math. Z. 48 (1943), 676-684.

17. F. Gonçalves, D. Oliveira e Silva, and S. Steinerberger, Hermite polynomials, linear flows on the torus, and an uncertainty principle for roots, J. Math. Anal. Appl. 451 (2017), no. 2, 678-711.

18. P. J. Grabner, Quasimodular forms as solutions of modular differential equations, Int. J. Number Theory (2020), to appear, available at https://arxiv.org/abs/2002.02736.

19. T. C. Hales, A proof of the Kepler conjecture, Ann. of Math. (2) 162 (2005), no. $3,1065-1185$.

20. H. Iwaniec, Topics in classical automorphic forms, Graduate Studies in Mathematics, vol. 17, American Mathematical Society, Providence, RI, 1997.

21. P. Jenkins and J. Rouse, Bounds for coefficients of cusp forms and extremal lattices, Bull. Lond. Math. Soc. 43 (2011), no. 5, 927-938.

22. G. A. Kabatjanskiŭ and V. I. Levenšteı̆n, Bounds for packings on the sphere and in space, Problemy Peredači Informacii 14 (1978), no. 1, 3-25.

23. M. Kaneko and M. Koike, On extremal quasimodular forms, Kyushu J. Math. 60 (2006), no. 2, 457-470.

24. M. Kaneko, K. Nagatomo, and Y. Sakai, The third order modular linear differential equations, J. Algebra 485 (2017), 332-352.

25. M. Kaneko and D. Zagier, A generalized Jacobi theta function and quasimodular forms, The moduli space of curves (Texel Island, 1994), Progr. Math., vol. 129, Birkhäuser Boston, Boston, MA, 1995, pp. 165-172.

26. R. E. A. C. Paley and N. Wiener, Fourier transformations in the complex domain, AMS Colloquium Publications, vol. XIX, American Mathematical Society, New York, 1934.

27. D. Radchenko and M. Viazovska, Fourier interpolation on the real line, Publ. Math. Inst. Hautes Études Sci. 129 (2019), 51-81.

28. L. Rolen and I. Wagner, A note on Schwartz functions and modular forms, Arch. Math. (2020), to appear.

29. E. Royer, Quasimodular forms: an introduction, Ann. Math. Blaise Pascal 19 (2012), no. 2, 297-306.

30. G. Shimura, Modular forms: basics and beyond, Springer Monographs in Mathematics, Springer, New York, 2012.

31. W. Stein, Modular forms, a computational approach, Graduate Studies in Mathematics, vol. 79, American Mathematical Society, Providence, RI, 2007, With an appendix by Paul E. Gunnells.

32. M. S. Viazovska, The sphere packing problem in dimension 8, Ann. of Math. (2) 185 (2017), no. 3, 991-1015.

33. D. V. Widder, The Laplace Transform, Princeton Mathematical Series, v. 6, Princeton University Press, Princeton, N. J., 1941.

34. T. Yamashita, On a construction of extremal quasimodular forms of depth two, Master's thesis, Tsukuba University, 2010, Japanese. 
35. D. Zagier, Modular forms and differential operators, Proc. Indian Acad. Sci. Math. Sci. 104 (1994), no. 1, 57-75, K. G. Ramanathan memorial issue.

36. Elliptic modular forms and their applications, in The 1-2-3 of modular forms [4, pp. 1-103.

(AF\&DH) Department of Mathematics, Vanderbilt University, 1326 Stevenson Center, Nashville, TN 37240, USA

E-mail address: ahram.s.feigenbaum@Vanderbilt.Edu

E-mail address: doug.hardin@Vanderbilt.Edu

(PG) Institute of Analysis and Number Theory, Graz University of Technology, Kopernikusgasse 24. 8010 Graz, Austria

E-mail address: peter.grabner@tugraz.at 